\title{
Passive Cascaded-Lattice Structures for Low-Sensitivity FIR Filter Design, with Applications to Filter Banks
}

\author{
P. P. VAIDYANATHAN, MEMBER, IEEE
}

\begin{abstract}
A class of nonrecursive cascaded-latlice struclures is derived. for the implementation of finite-impulse response (FIR) digital filters. The building blocks are lossless and the transfer function can be implemented as a sequence of planar rotations. The structures can be used for the synthesis of any scalar FIR transfer function $H(z)$ with no restriction on the location of zeros; at the same time, all the lattice coefficients have magnitude bounded above by unity. The structures have excellent passband sensitivity because of inherent passivity, and are automatically internally scaled, in an $L_{2}$ sense.

The ideas are also extended for the realization of a bank of $M$ FIR transfer functions as a cascaded lattice. Applications of these structures in subband coding and in multirate signal processing are outlined. Numerical design examples are included.
\end{abstract}

\section{INTRODUCTION}

$\mathrm{T}$ HERE EXIST A number of methods [1]-[8] for the design of low-sensitivity infinite impulse response (IIR) digital filters. Notable among these are the wellknown wave-digital filters [1], [2], orthogonal digital filters [4], and certain types of lattice filters [6], [7]. Some of these methods are based on the notion of pseudopassivity, while certain others are based on orthogonality of internal computations. The lattice digital filters reported thus far can also be related to autoregressive/moving average modeling techniques [9]-[11]. The relation between these families of filters has also been known for some time [14], [24], [25], [28].

In principle, any stable IIR digital filtcr transfer function can be implemented as an orthogonal filter [4], [5], [14], with planar rotation building blocks. The purpose of this paper is to develop passive structures for arbitrary finite impulse response (FIR) transfer functions and FIR filter banks based on planar rotation building blocks. An important feature of many of the well-known IIR digital filter structures having favorable finite-wordlength properties is that some or all of the internal building blocks are passive in a certain sense, and this has been favorably exploited [7], [12], [13] to obtain low-sensitivity designs free of limit cycles. A normalized version of the passivitybased structures results in orthogonal implementations [6],

Manuscript received July 2, 1985; revised June 6, 1986.

This work was supported in part by the National Science Foundation under Grant ECS 84-04245 and in part by Caltech's program in Advanced Technology sponsored by Aerojet General, General Motors, GTE, and TRW.

The author is with the Department of Electrical Engineering, California Institute of Technology, Pasadena, CA 91125.

IEEE Log Number 8610176.
[14] for IIR filters, which have the additional advantage that all internal signals are scaled in the $l_{2}$ sense, thus rendering additional scaling effort unnecessary.

When implementing FIR digital filters, there is clearly no possibility of limit cycles, as long as the structure is nonrecursive. ${ }^{1}$ However, it is still of interest to obtain structures that have low sensitivity. It is also desirable to have normalized structures, which in addition have low noise.

Schuessler [15], [16] has studied quantization effects in FIR filters, and a variety of interesting structures based on polynomial-interpolation theory can be found in [16]. Chan and Rabiner [17], [18] have done extensive research on cascade-form FIR filters, with particular emphasis on the ordering of the sections to attain optimal noise/dynamic range performance. The general conclusion seems to be that direct-form implementations of FIR filters have poor stopband sensitivity while cascade-form realizations have reasonably good stopband sensitivity, even though the passband behavior tends to deteriorate when the multiplier coefficients are quantized. Moreover, unless the cascading order is properly chosen, the roundoff noise gain can be excessively large.

A class of FIR lattice digital filters has been studied by Makhoul and others [10], [11], [21] in the context of linear-predictive coding of signals. A subset of these structures naturally arises while solving the "normal equations" via Levinson's recursion. A main feature of these structures is that, as long as the multiplier coefficients $k_{l}$ (called the "reflection" coefficients) are bounded (in magnitude) by unity, a pair of FIR transfer functions $G(z)$ and $H(z)$ can be realized where $G(z)$ has minimum phase and $H(z)$ has maximum phase. However, with $k_{l}^{2}<1$, one cannot realize arbitrary transfer functions, for example, an equiripple FIR filter with some of its zeros on the unit circle of the z-plane. (It is, however, easy to force all zeros to be on the unit circle [22] simply by setting the "rightmost $k_{l}$ " to \pm 1 .)

The FIR lattice filters arising out of linear-predictive coding of signals have an IIR counterpart, typically called the "synthesis filters" [11]. The IIR or recursive counterpart, also known as the Gray-and-Markel filter structure

\footnotetext{
${ }^{1}$ Recursive realizations of FIR filters, such as the frequency-sampling structures, can, theoretically support limit cycles.
} 
[6], [7], has several equivalent forms, one of them being the "normalized" lattice form [6]. These IIR structures are known to have passivity properties [7], and in addition, the normalized lattice is based on a cascade of orthogonal (planar rotation) building blocks. The IIR structures have excellent "robustness" properties under quantized conditions. Even though this IIR version has passivity properties, the FIR counterpart does not (this will be elaborated on in Section V).

The FIR lattice structures we introduce in this paper are not related to linear-predictive coding in the sense that the "corresponding" IIR versions do not necessarily represent a maximum-entropy spectral model [11] of any appropriate time series. The new FIR structures are characterized by a set of coefficients $k_{l}$ such that $k_{l}^{2} \leqslant 1$, yet any arbitrary FIR transfer function $H(z)$ with no restriction on the zero locations can be realized. (Accordingly, the "corresponding" IIR version does not necessarily represent a stable filter.) These FIR structures are based on simple interconnections of orthogonal (planar rotation) building blocks, and are "passive" in the same sense (to be elaborated on) as the well-known orthogonal and wave-digital IIR filters. They are automatically internally scaled and have low passband sensitivity. In spite of the fact that they are in the form of a nonrecursive cascade, we do not encounter serious roundoff noise problems. Indeed, the noise-variance gain is bounded above by the filter order, exactly as in the direct-form structure [19], [20]. Finally, these structures seem to be a natural choice for certain types of filter banks and multirate subband coding applications involving"quadrature mirror filters" [23].

In Section II, we briefly review the structural-passivity concept and its relation to low sensitivity. Section III introduces the new FIR lossless lattice structures. A synthesis procedure is presented in this section so that an arbitrary FIR transfer function (not necessarily linear phase) can be implemented in this form. The result of synthesis is a one-input two-output structure, with transfer functions $G(z)$ and $H(z)$, where $G(z)$ is the desired FIR function and $H(z)$ is an auxiliary function such that $\left|G\left(e^{j \omega}\right)\right|^{2}+\left|H\left(e^{j \omega}\right)\right|^{2}=1$. We thus have a bank of two filters. Section IV is a study of certain important properties of this class of structures. Next, in Section V, we review the relation between the new structures and certain well-known lattice structures for digital filtering. In Section VI, we extend the idea of Section III to the case of a filter bank of $M$ FIR transfer functions $G_{0}(z)$, $G_{1}(z), \cdots, G_{M-1}(z)$ realized as a cascaded lossless FIR lattice structure. The bank is such that $\left|G_{0}\left(e^{j \omega}\right)\right|^{2}+\cdots$ $\left|G_{M-1}\left(e^{j \omega}\right)\right|^{2}=1$. Section VII presents a general roundoff-noise/dynamic range analysis, which applies to all structures presented in the paper. In Section VIII, applications in analysis/synthesis filter banks are presented. Finally, in Section IX, we present an application of the structures in multirate digital filtering, where sampling-rate changes are involved in addition to linear time-invariant filtering.
Notations Used in the Sequel: In this paper, superscript $t$ stands for matrix transposition, whereas superscript dagger $(\dagger)$ stands for transposition followed by complex conjugation. Bold-faced letters indicate vectors and matrices. The tilde accent stands for transposition followed by reciprocation of functional argument; for example, $\tilde{H}(z)=H^{t}\left(z^{-1}\right)$. The notation $\boldsymbol{A} \leqslant \boldsymbol{B}$ (where $\boldsymbol{A}$ and $\boldsymbol{B}$ are square matrices of equal dimensions) is abbreviation for " $\boldsymbol{B}-\boldsymbol{A}$ is positive semidefinite." Similarly, $\boldsymbol{A}<\boldsymbol{B}$ means " $\boldsymbol{B}-\boldsymbol{A}$ is positive definite." $\boldsymbol{I}_{m}$ (with subscript possibly omitted) denotes the identity matrix of dimension $m \times m$. For a (real symmetric) positive definite matrix $\boldsymbol{P}$, we define its square root $\boldsymbol{P}^{1 / 2}$ according to the factorization $\boldsymbol{P}=\boldsymbol{P}^{1 / 2} \boldsymbol{P}^{t / 2}$, where $\boldsymbol{P}^{t / 2}$ is the transpose of $\boldsymbol{P}^{1 / 2}$.

\section{Structural Boundedness AND LOW SENSITIVITY}

A stable digital filter transfer function $H(z)$ with real coefficients is said to be bounded real (BR) if

$$
\left|H\left(e^{j \omega}\right)\right| \leqslant 1, \quad \text { for all } \omega .
$$

If equality holds in (1) for all $\omega$, then $H(z)$ is said to be lossless bounded real (LBR). An implementation is said to be structurally passive or structurally bounded [24] if, regardless of the multiplier values (as long as they are in a well-defined range such as $-1 \leqslant m_{k} \leqslant 1$ ), the transfer function satisfies (1). If the multiplier values in a structurally passive implementation are such that $\left|H\left(e^{j \omega}\right)\right|$ attains the upper bound of unity in the passband(s), then the structure has low passband sensitivity [14], [24]. It is useful to extend these definitions to the matrix case. A stable transfer matrix $\boldsymbol{H}(z)$ with real coefficients is BR if $\boldsymbol{H}^{\dagger}\left(e^{j \omega}\right) \boldsymbol{H}\left(e^{j \omega}\right) \leqslant \boldsymbol{I}$ for all $\omega$, and LBR (or "allpass") if this inequality becomes an equality for all $\omega$.

Given an FIR BR transfer function $H(z)$ of order $N$ satisfying (1), we can always find another FIR BR function $G(z)$ such that

$$
\left|G\left(e^{j \omega}\right)\right|^{2}+\left|H\left(e^{j \omega}\right)\right|^{2}=1, \quad \text { for all } \omega .
$$

The vector $G_{N}(z)=\left[\begin{array}{ll}H(z) & G(z)\end{array}\right]^{t}$ of order $N$ is, hence, FIR LBR (or "allpass"), and we show how this FIR vector can be realized in terms of FIR lossless building blocks. Such a realization exhibits low sensitivity in the passbands of both $G(z)$ and $H(z)$. This idea of embedding $H(z)$ into a vector $G_{N}(z)$ is easily generalized. Thus, given $M-1$ FIR BR transfer functions $G_{0}(z), G_{1}(z), \cdots, G_{M-2}(z)$ such that

$$
\left|G_{0}\left(e^{j \omega}\right)\right|^{2}+\left|G_{1}\left(e^{j \omega}\right)\right|^{2}+\cdots+\left|G_{M-2}\left(e^{j \omega}\right)\right|^{2} \leqslant 1
$$

for all $\omega$, we can always find an FIR BR function $G_{M-1}(z)$ such that the vector $\boldsymbol{G}_{N}(z)=\left[G_{0}(z), G_{1}(z), \cdots, G_{M-1}(z)\right]^{t}$ is FIR LBR (or “allpass”).

\section{LATTICE STRUCTURES FOR TWO-COMPONENT FIR Allpass VeCtoRS}

Let us assume that we have an FIR allpass vector

$$
\boldsymbol{G}_{N-1}(z)=\left[\begin{array}{ll}
P_{N-1}(z) & Q_{N-1}(z)
\end{array}\right]^{t}
$$




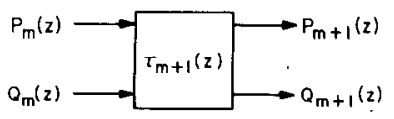

Fig. 1. Realization of $\boldsymbol{G}_{m+1}(z)$.

where

$$
\begin{aligned}
P_{N-1}(z) & =\sum_{n=0}^{N-1} p_{N-1, n} z^{-n} \\
Q_{N-1}(z) & =\sum_{n=0}^{N-1} q_{N-1, n} z^{-n}
\end{aligned}
$$

It is possible that one of them, say, $P_{N-1}(z)$, has linear phase. However, in view of the allpass property

$$
\tilde{P}_{N-1}(z) P_{N-1}(z)+\tilde{Q}_{N-1}(z) Q_{N-1}(z)=1
$$

it is not possible for both $P_{N-1}(z)$ and $Q_{N-1}(z)$ to have linear phase, except in some trivial situations [31]. We wish to synthesize $G_{N-1}(z)$ as a nonrecursive cascaded-lattice structure. The basic step in the synthesis procedure is the order-reduction problem, which we address next.

\section{A. The Order-Reduction Problem}

Let $G_{m+1}(z)=\left[\begin{array}{ll}P_{m+1}(z) & Q_{m+1}(z)\end{array}\right]^{t}$ be an $(m+1)$ thorder FIR allpass vector

$$
\begin{gathered}
P_{m+1}(z)=\sum_{n=0}^{m+1} p_{m+1, n} z^{-n} \\
Q_{m+1}(z)=\sum_{n=0}^{m+1} q_{m+1, n} z^{-n} \\
\tilde{P}_{m \backslash 1}(z) P_{m+1}(z)+\tilde{Q}_{m+1}(z) Q_{m+1}(z)=1 .
\end{gathered}
$$

We wish to realize $\boldsymbol{G}_{m+1}(z)$ in the form shown in Fig. 1, where $\tau_{m+1}(z)$ is a first-order $2 \times 2$ FIR allpass matrix, and where $G_{m}(z)=\left[P_{m}(z) \quad Q_{m}(z)\right]^{t}$ is a lower order FIR allpass vector

$$
\begin{gathered}
P_{m}(z)=\sum_{n=0}^{m} p_{m, n} z^{-n} \\
Q_{m}(z)=\sum_{n=0}^{m} q_{m, n} z^{-n} . \\
\tilde{P}_{m}(z) P_{m}(z)+\tilde{Q}_{m}(z) Q_{m}(z)-1 .
\end{gathered}
$$

Repeated application of the order-reduction process results in the desired cascaded-lattice realization. In order to accomplish this order reduction,we note that the allpass property of (7) implies, in particular,

$$
p_{m+1,0} p_{m+1, m+1}+q_{m+1,0} q_{m+1, m+1}=0 .
$$

If $p_{m+1, m+1}$ turns out to be zero, then (10) implies either $q_{m+1, m+1}=0$ (in which case, the current order-reduction step is not necessary and $\tau_{m+1}=I$ ) or $q_{m+1,0}=0$, in which case we get

$$
\begin{aligned}
P_{m}(z) & =P_{m+1}(z) \\
Q_{m}(z) & =z Q_{m+1}(z) \\
& =q_{m+1,1}+q_{m+1,2} z^{-1}+\cdots+q_{m+1, m+1} z^{-m} \\
\tau_{m+1}(z) & =\left[\begin{array}{cc}
1 & 0 \\
0 & z^{-1}
\end{array}\right] .
\end{aligned}
$$

Having taken care of these simple situations, let us now assume that none of the four quantities in the left-hand side of $(10)$ is zero. Define

$$
P_{m}(z)=k_{m+1} P_{m+1}(z)+\hat{k}_{m+1} Q_{m+1}(z)
$$

where

$$
\begin{aligned}
& k_{m+1}=\frac{-q_{m+1, m+1}}{\sqrt{p_{m+1, m+1}^{2}+q_{m+1, m+1}^{2}}} \\
& \hat{k}_{m+1}=\frac{p_{m+1, m+1}}{\sqrt{p_{m+1, m+1}^{2}+q_{m+1, m+1}^{2}}} .
\end{aligned}
$$

Clearly, with the above choice, the highest power, i.e., $z^{-(m+1)}$ cancels on the right-hand side of (12). Now consider the quantity

$$
-\hat{k}_{m+1} P_{m+1}(z)+k_{m+1} Q_{m+1}(z) .
$$

In view of (10) and the choices of $k_{m+1}$ and $\hat{k}_{m+1}$, we have

$$
-\hat{k}_{m+1} p_{m+1,0}+k_{m+1} q_{m+1,0}=0 .
$$

Hence, the constant term in (15) is zero. In other words, if we force the highest term on the right-hand side of (12) to zero, then the lowest term in (15) is automatically zero because of the LBR property of $\boldsymbol{G}_{m+1}(z)$. Accordingly, if we define $\boldsymbol{G}_{m}(z)=\left[\begin{array}{ll}P_{m}(z) & Q_{m}(z)\end{array}\right]^{t}$ by

$$
\boldsymbol{G}_{m}(z)=\left[\begin{array}{c}
P_{m}(z) \\
Q_{m}(z)
\end{array}\right]=\left[\begin{array}{cc}
k_{m+1} & \hat{k}_{m+1} \\
-\hat{k}_{m+1} z & k_{m+1} z
\end{array}\right]\left[\begin{array}{c}
P_{m+1}(z) \\
Q_{m+1}(z)
\end{array}\right]
$$

with $k_{m+1}$ and $\hat{k}_{m+1}$ as in (13) and (14), then $P_{m}(z)$ and $Q_{m}(z)$ indeed have lower order. Moreover, from (17)

$$
\begin{aligned}
\boldsymbol{G}_{m+1}(z) & \triangleq\left[\begin{array}{l}
P_{m+1}(z) \\
Q_{m+1}(z)
\end{array}\right] \\
& =\left[\begin{array}{cc}
k_{m+1} & -\hat{k}_{m+1} z^{-1} \\
\hat{k}_{m+1} & k_{m+1} z^{-1}
\end{array}\right]\left[\begin{array}{l}
P_{m}(z) \\
Q_{m}(z)
\end{array}\right]
\end{aligned}
$$

i.e.,

$$
\boldsymbol{G}_{m+1}(z)=\tau_{m+1}(z) \boldsymbol{G}_{m}(z)
$$

where the matrix

$$
\tau_{m+1}(z)=\left[\begin{array}{cc}
k_{m+1} & -\hat{k}_{m+1} z^{-1} \\
\hat{k}_{m+1} & k_{m+1} z^{-1}
\end{array}\right]
$$

satisfies $\tilde{\tau}_{m+1} \tau_{m+1}=I$ and is, hence, LBR. Equation (19) 


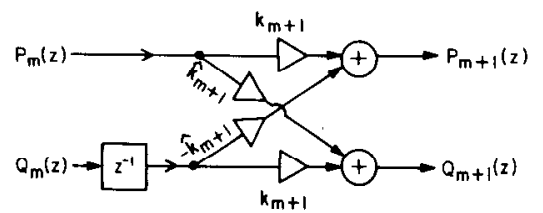

Fig. 2. Implementation of $G_{m+1}(z)$ from $G_{m}(z)$.

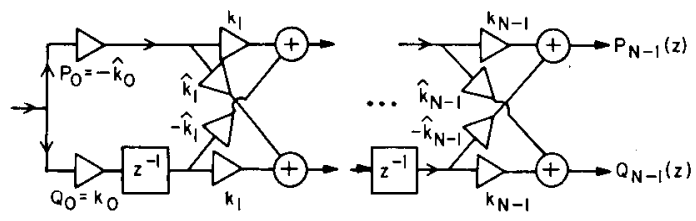

Fig. 3. The resulting cascaded-lattice structure.

therefore implies

$$
\begin{aligned}
1 & =\tilde{\boldsymbol{G}}_{m+1}(z) \boldsymbol{G}_{m+1}(z)=\tilde{\boldsymbol{G}}_{m}(z) \tilde{\tau}_{m+1}(z) \tau_{m+1}(z) \boldsymbol{G}_{m}(z) \\
& =\tilde{\boldsymbol{G}}_{m}(z) \boldsymbol{G}_{m}(z)
\end{aligned}
$$

which shows that $\boldsymbol{G}_{m}(z)$ is indeed lower order FIR allpass. This then completes the order reduction problem.

Fig. 2 shows the implementation of $\boldsymbol{G}_{m+1}(z)$, in terms of $\boldsymbol{G}_{m}(z)$ and $\tau_{m+1}(z)$. Fig. 3 shows the cascaded-lattice structure that results by repeated application of the above process by starting with the FIR allpass function $\boldsymbol{G}_{N-1}(z)$ $=\left[\begin{array}{ll}P_{N-1}(z) & Q_{N-1}(z)\end{array}\right]^{t}$. Equation (17), which represents the "downward recursion" can also be rewritten as

$$
\left[\begin{array}{c}
P_{m}(z) \\
-Q_{m}(z)
\end{array}\right]=\left[\begin{array}{cc}
k_{m+1} & \hat{k}_{m+1} \\
\hat{k}_{m+1} z & -k_{m+1} z
\end{array}\right]\left[\begin{array}{c}
P_{m+1}(z) \\
Q_{m+1}(z)
\end{array}\right]
$$

and this leads to a lattice section with minor difference in internal details.

Equation (17), which enables us to synthesize the structure starting from $\boldsymbol{G}_{N-1}(z)$, is called the downwards recursion scheme, whereas (18), which enables us to compute $\boldsymbol{G}_{N-1}(z)$ starting from a set of values $k_{0}, k_{1}, \cdots, k_{N-1}$, is called the upwards recursion.

\section{B. Properties of the Cascaded FIR Lattice Structure}

1) It follows from (13) and (14) that the structure of Fig. 3 has $^{2} k_{m}^{2} \leqslant 1$ for all $m$, and moreover $k_{m}^{2}+\hat{k}_{m}^{2}=1$. Thus, the cascade shown in Fig. 3 is made of "building blocks" that are planar rotation operators, with successive building blocks being separated by a delay. The structure is therefore highly modular, and moreover only adjacent building blocks are directly connected. Notice that each planar rotation is equivalent to a complex-multiplication operation, with real and imaginary parts of operands and results defined in an obvious manner.

2) Given any FIR filter transfer function $P_{N-1}(z)$ with no restrictions on the zero locations, we can always obtain an implementation as in Fig. 3 with $k_{m}^{2} \leqslant 1$, as long as $\left|P_{N-1}\left(e^{j \omega}\right)\right|^{2} \leqslant 1$.

3) The structure of Fig. 3 can also be looked upon as the interconnection of $2 \times 2$ LBR structures with transfer

\footnotetext{
${ }^{2}$ If $k_{m}^{2}=0$ or 1 , we call the corresponding section in Fig. 3 "degener-
}
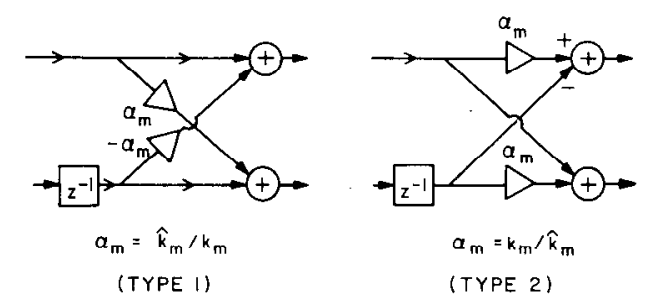

Fig. 4. Denormalized lattice sections requiring only two multipliers The choice of the type should be made such that $\alpha_{m}^{2}$ is less than unity.

matrices as in (20). Accordingly, we call the structure a cascaded normalized lattice structure, or a cascaded lossless lattice structure.

4) Because of the allpass property of $\boldsymbol{G}_{m+1}(z),(10)$ holds, which gives us an alternative expression for $k_{m+1}$ and $\hat{k}_{m+1}$ of (13) and (14)

$$
k_{m+1}=\frac{p_{m+1,0}}{\sqrt{p_{m+1,0}^{2}+q_{m+1,0}^{2}}} \quad \hat{k}_{m+1}=\frac{q_{m+1,0}}{\sqrt{p_{m+1,0}^{2}+q_{m+1,0}^{2}}} .
$$

Substitution of (23) into (12) reveals that, in view of (10), the term corresponding to the highest power $z^{-m-1}$ cancels in (12). Accordingly, the signs of $k_{m+1}$ and $\hat{k}_{m+1}$ are those of $p_{m+1,0}$ and $q_{m+1,0}$. In fact, only the relative signs of $k_{m+1}$ and $\hat{k}_{m+1}$ matters in the order-reduction process.

5) Unless each building block is implemented as a planar rotation, the total number of multipliers per section is four. Thus, a pair of $(N-1)$ th-order FIR filters $P_{N-1}(z)$ and $Q_{N-1}(z)$ requires $4(N-1)+2$ multipliers, i.e., $(2 N-1)$ per transfer function. Recall that the direct-form implementation requires only $N$ multipliers (and only about $\mathrm{N} / 2$ for linear-phase filters). The increased multiplier overhead can be explained by observing that the normalized building blocks render it unnecessary to perform internal signal-scaling in the structure of Fig. 3. (Normalized realizations always have multiplier-overhead [4]-[6].) Moreover, the structure of Fig. 3 has low passband sensitivity as expected from the structural-boundedness property and as demonstrated in later sections.

It is possible to obtain "denormalized lattice-sections" as shown in Fig. 4. This structure is particularly feasible when the values of $k_{m}^{2}$ or $\hat{k}_{m}^{2}$ are reasonably close to unity, as happens in most digital filtering examples. Notice that the multipliers $\alpha_{m}$ in the denormalized sections continue to satisfy $\left|\alpha_{m}\right| \leqslant 1$. The denormalized structure requires $N+1$ multipliers per transfer function. For filters with nonlinear phase, this is the same as for a direct-form implementation. For structures with linear phase, it is shown in Section IV that the lattice coefficients have a symmetry, i.e., $k_{m}= \pm k_{N-1-m}$; however, it is not clear how this can be exploited to reduce the number of multipliers by a factor of two.

\section{Design Example 1}

Consider an example where $P_{N-1}(z)$ is a linear-phase low-pass equiripple FIR filter, designed with the McClellan-Parks algorithm [27] to meet the following 
TABLE I

LATTICE COEfFicients for EXAMPLe 1

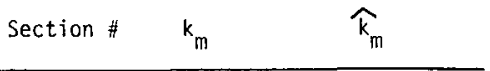

$0.002331 \quad 0.999997$

$0.999871 \quad 0.016038$

$0.999939 \quad 0.011081$

$0.999974 \quad 0.007208$

$-1.000000 \quad 0$.

- $0.999956 \quad 0.009332$

$-0.999894 \quad 0.014533$

$\begin{array}{ll}-0.999927 & 0.012089\end{array}$

$-0.999999 \quad 0.001686$

$0.999926 \quad 0.012183$

$0.999762 \quad 0.021803$

$\begin{array}{ll}0.999762 & 0.021803 \\ 0.999793 & 0.020337\end{array}$

$\begin{array}{ll}0.999793 & 0.020337 \\ 0.999981 & 0.006167\end{array}$

$-0.999889 \quad 0.014912$

$-0.999505 \quad 0.031474$

$-0.999481 \quad 0.032228$

$-0.999915 \quad 0.013047$

0.999824

0.999824

0.998941

$0.998748 \quad 0.050015$

$0.999760 \quad 0.021926$

$\begin{array}{ll}-0.999587 & 0.028726\end{array}$

$-0.997249 \quad 0.074126$

$-0.996791$

$-0.999647$

$\begin{array}{ll}0.999647 & 0.026561 \\ 0.997416 & 0.071841\end{array}$

$0.987856 \quad 0.155375$

$0.991294 \quad 0.131669$

$-0.995787 \quad 0.091698$

$-0.867826 \quad 0.496868$

$-0.713467 \quad 0.700689$

specifications: passband edge $\omega_{p}=0.196 \pi$, stopband edge $\omega_{s}=0.27 \pi$, passband peak ripple $\delta_{1} \leqslant 0.0013$, stopband attenuation $A_{s} \geqslant 32 \mathrm{~dB}$. The desired filter order turns out to be $N-1=60$. Once the BR transfer function $P_{N-1}(z)$ is obtained, $Q_{N-1}(z)$ can be found by solving for a spectral factor $\left[1-\left|P_{N-1}\left(e^{j \omega}\right)\right|^{2}\right]$. Mian and Nainer [26] have outlined a procedure for computing spectral factors without going through tedious root-finding procedures. The method outlined in [26] works even if there are zeros on the unit circle, and is based on the computation of the complex cepstrum of a suitably modified sequence that does not have zeros on the unit circle.

Once $P_{N-1}(z)$ and $Q_{N-1}(z)$ are obtained, the lattice structure of Fig. 3 can be synthesized as described earlier. Notice that $Q_{N-1}(z)$ does not have linear phase. The lattice coefficients $k_{m}$ and $\hat{k}_{m}$ are tabulated in 'Table I for $0 \leqslant m \leqslant 30$. In view of the linear phase nature of $P_{N-1}(z)$, the lattice coefficients $k_{m}$ satisfy a symmetry property (see (36)) as shown in Section IV. Accordingly, we have not listed $k_{31}, \cdots, k_{60}$. Notice that a number of $k$-parameters have magnitude close to unity, and an unscaled version of the corresponding lattice sections can be used without seriously affecting internal scaling. Fig. 5 shows the magnitude responses $\left|P_{N-1}\left(e^{j \omega}\right)\right|$ and $\left|Q_{N-1}\left(e^{j \omega}\right)\right|$ of the simulated FIR lattice of Fig. 3

In order to demonstrate the low passband-sensitivity properties of the cascaded FIR lattice structures, the denormalized lattice structure for the above pair of transfer functions has been simulated, with coefficients quantized to three binary bits in canonic sign-digit code. More specifically, each multiplier is approximated by a sum of

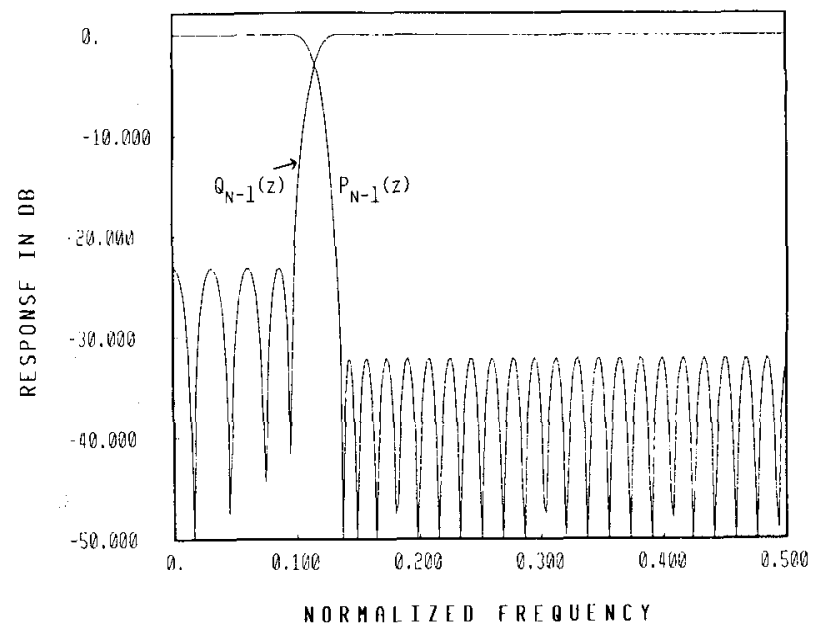

Fig. 5. Example 1. Magnitude response in decibels for a simulated LBR lattice.

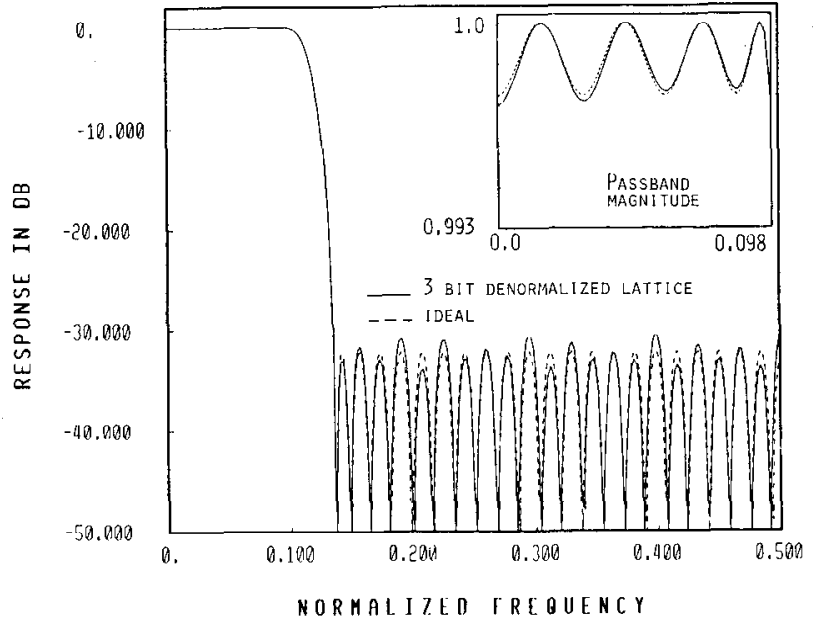

(a)

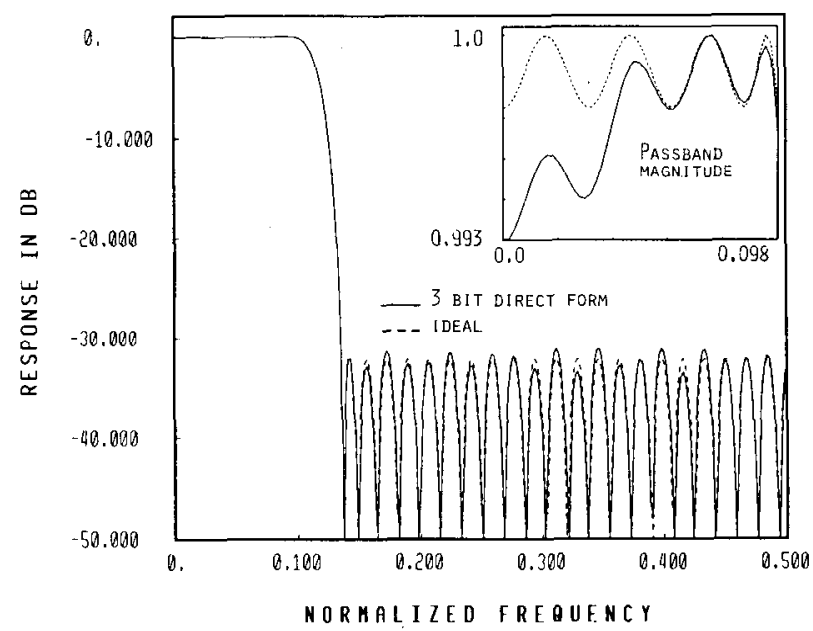

(b)

Fig. 6. (a) Example 1. The simulated lattice response with 3 bits per multiplier (in this paper $b$-bits per multiplier means that the multiplier is approximated with $b$ powers of two). (b) Example 3.1. The direct-form structure with 3 bits per multiplier. 

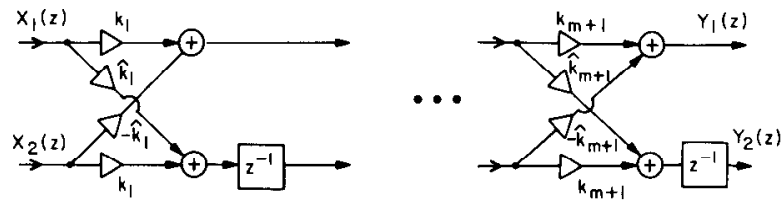

Fig. 7. Pertaining to properties of the cascaded lattice

three powers of two. The resulting frequency response magnitude $\left|P_{N-1}\left(e^{j \omega}\right)\right|$ is shown in Fig. 6 for the cascaded FIR lattice structures, and for a conventional direct-form implementation quantized in the same manner. Even though the direct form has somewhat better stopband performance, the passband details shown in Fig. 6(a) clearly demonstrate the excellent passband behavior of the lattice structure.

\section{AdDitional Properties of THE FIR Cascaded-Lattice Structures}

In this section, we establish a few more interesting properties of the cascaded lossless lattice structures developed in Section III. For simplicity, we assume that none of the $k$-parameters is "degenerate," i.e., $0 \neq k_{m}^{2} \neq 1$ for any $m$. At the expense of additional notational complexity, all the properties we wish to study can be restated when this assumption is not true. Let us first consider the cascade shown in Fig. 7, which represents a cascade of $m+1$ LBR sections.

Property 1: Let $\tau(z)=\left[T_{i j}\right]$ represent the LBR transfer matrix of this overall cascade, i.e., let

$$
\left[\begin{array}{l}
Y_{1}(z) \\
Y_{2}(z)
\end{array}\right]=\left[\begin{array}{ll}
T_{11}(z) & T_{12}(z) \\
T_{21}(z) & T_{22}(z)
\end{array}\right]\left[\begin{array}{l}
X_{1}(z) \\
X_{2}(z)
\end{array}\right] .
$$

Then its elements are related as follows

$$
\begin{aligned}
& T_{11}(z)=z^{-(m+1)} T_{22}\left(z^{-1}\right) \\
& T_{12}(z)=-z^{-(m+1)} T_{21}\left(z^{-1}\right) .
\end{aligned}
$$

For the case of $m=0,(25)$ can be explicitly verified. Next, assuming that the relation of this form is true for a cascade of $m$ sections, it can be shown that it continues to be true if a new section is added to the cascade. In this manner, (25) can be inductively established; details are omitted.

Property 2: In the cascade of Fig. 7, let $T_{12}(z)$ be a linear phase FIR transfer function. Then the following is

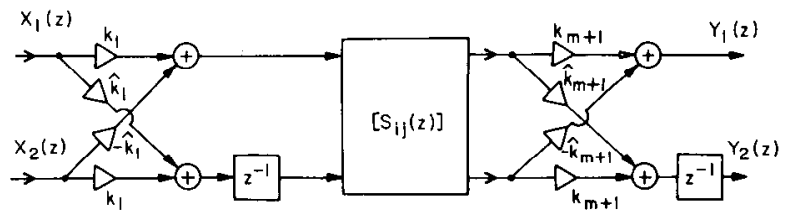

Fig. 8. Pertaining to symmetry properties.

Because of the linear-phase property of $T_{12}(z)$, we have the following symmetry relation:

$$
T_{12, k}=\Delta \cdot T_{12, m-k}, \quad 0 \leqslant k \leqslant m
$$

where $\Delta$ is a constant equal to 1 or -1 . Recall our assumption that none of the lattice sections is degenerate. Accordingly, $0 \neq k_{I}^{2} \neq 1$ in (28), and we have, by (28) and (29) $\hat{k}_{1} k_{m+1}=\Delta \cdot k_{1} \hat{k}_{m+1}$, which on simplification leads to (26).

The next property is concerned with the behavior of internal transfer functions. Let us redraw Fig. 7 in the form of Fig. 8. The transfer matrix $\left[S_{i j}(z)\right]$ is defined according to

$$
\left[\begin{array}{l}
Y_{1}^{\prime}(z) \\
Y_{2}^{\prime}(z)
\end{array}\right]=\left[\begin{array}{ll}
S_{11}(z) & S_{12}(z) \\
S_{21}(z) & S_{22}(z)
\end{array}\right]\left[\begin{array}{l}
X_{1}^{\prime}(z) \\
X_{2}^{\prime}(z)
\end{array}\right]
$$

whereas $T_{i j}(z)$ are defined according to (24).

Property 3: If $T_{12}(z)$ has linear phase, then $S_{12}(z)$ has linear phase.

Proof: In order to show. this, first recall that the $T_{i j}(z)$ parameters satisfy (25), whereas the parameters $S_{i j}(z)$ satisfy

$$
\begin{aligned}
& S_{11}(z)=z^{-(m-1)} S_{22}\left(z^{-1}\right) \\
& S_{12}(z)=-z^{-(m-1)} S_{21}\left(z^{-1}\right) .
\end{aligned}
$$

Next, from Fig. 8 it is clear that

$$
\begin{aligned}
{\left[\begin{array}{ll}
S_{11}(z) & S_{12}(z) \\
S_{21}(z) & S_{22}(z)
\end{array}\right]=} & {\left[\begin{array}{cc}
k_{m+1} & -\hat{k}_{m+1} \\
\hat{k}_{m+1} z^{-1} & k_{m+1} z^{-1}
\end{array}\right]^{-1} } \\
& \cdot\left[\begin{array}{cc}
T_{11}(z) & T_{12}(z) \\
T_{21}(z) & T_{22}(z)
\end{array}\right] \\
& \cdot\left[\begin{array}{cc}
k_{1} & -\hat{k}_{1} \\
\hat{k}_{1} z^{-1} & k_{1} z^{-1}
\end{array}\right]^{-1}
\end{aligned}
$$

from which we obtain

$$
S_{12}(z)=\frac{\left[\hat{k}_{1} k_{m+1} z^{-1} T_{11}(z)+k_{1} \hat{k}_{m+1} T_{22}(z)+k_{1} k_{m+1} z^{-1} T_{12}(z)+\hat{k}_{1} \hat{k}_{m+1} T_{21}(z)\right]}{z^{-2}} .
$$

true:

$$
k_{1}^{2}=k_{m+1}^{2}
$$

Proof: Denote the polynomial $T_{12}(z)$ by

$$
T_{12}(z)=T_{12,0}+T_{12,1} Z^{-1}+\cdots+T_{12, m} z^{-m} .
$$

Inspection of Fig. 7 reveals that

$$
\begin{aligned}
& T_{12,0}=-\hat{k}_{1} k_{2} k_{3} \cdots k_{m+1} \\
& T_{12, m}=-k_{1} k_{2} k_{3} \cdots \hat{k}_{m+1} .
\end{aligned}
$$

Recall that (25) and (31) are true; moreover, since $T_{12}(z)$ has linear phase, (29) holds, and $\hat{k}_{1} k_{m+1}=\Delta \cdot k_{1} \hat{k}_{m+1}$ holds. It can therefore be verified by explicit substitution in (33) that

$$
z^{-(m-2)} S_{12}\left(z^{-1}\right)=\Delta \cdot S_{12}(z)
$$

which shows that $S_{12}(z)$ has linear phase as well.

Property 4: In Fig. 7, if $T_{12}(z)$ has linear phase, then we have

$$
\begin{aligned}
\hat{k}_{1} k_{m+1} & =\Delta \cdot k_{1} \hat{k}_{m+1}, \hat{k}_{2} k_{m}=\Delta \cdot k_{2} \hat{k}_{m}, \hat{k}_{3} k_{m-1} \\
& =\Delta \cdot k_{3} \hat{k}_{m-1}, \cdots .
\end{aligned}
$$




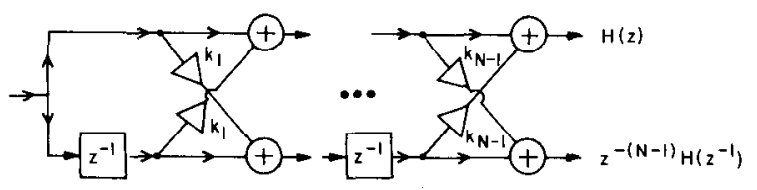

Fig. 9. The FIR filter structure related to linear-predictive coding.

Accordingly, $k_{1}^{2}=k_{m+1}^{2}, k_{2}^{2}=k_{m}^{2}, \cdots$, and so on. This property follows merely by applying Properties 2 and 3 repeatedly to the cascaded structure of Fig. 7.

The main consequence of these properties is the following: in the structure of Fig. 3, if $P_{N-1}(z)$ is a linear-phase transfer function, then the following is true:

$$
k_{l}^{2}=k_{N-1-l}^{2}, \quad 0 \leqslant l \leqslant N-1 .
$$

\section{Relation to Other Known Digital Filter STRUCTURES}

The denormalized structure of Fig. 4 immediately reveals the graphical similarity of the new lattice filters to the well-known FIR filter lattice structures arising in linear-prediction theory (reproduced in Fig. 9) [10], [11], [21]. However, the main difference is the appearance of a negative sign in each section of Fig. 4, unlike in linear-prediction FIR filters. This difference makes it possible for the circuit to realize arbitrary FIR transfer functions (within a scale factor) with arbitrary magnitude and phase responses.

The building blocks represented by (20) are themselves well known in the implementation of IIR orthogonal filters and IIR lattice digital filters [6], [14]. The normalized nature of these building blocks is primarily responsible for several excellent finite-wordlength properties of IIR orthogonal and lattice filters. Similar properties are inherited by the FIR structures of Fig. 3, as claborated on later.

It is well known that the FIR filter of Fig. 9 arising in lincar prediction is associated with a "corresponding" IIR digital filter, as shown in Fig. 10. The values of the corresponding $k$-paramcters are the same in Figs. 9 and 10. The transfer functions $H(z)$ and $z^{-(N-1)} H\left(z^{-1}\right)$ in Fig. 9 are related to the transfer functions $G_{1}(z)$ and $G_{2}(z)$ of Fig. 10 by

$$
G_{1}(z)=1 / H(z) \quad G_{2}(z)=z^{-(N-1)} H\left(z^{-1}\right) / H(z) .
$$

Thus, $G_{1}(z)$ is an "all-pole" function, whereas $G_{2}(z)$ is allpass. The normalized version of the structure of Fig. 10, known as the normalized Gray-and-Markel IIR cascaded lattice, is shown in Fig. 11. Notice that the basic building blocks in the IIR structure of Fig. 11 have the same transfer matrix as the FIR filter building blocks in Fig. 3.

The fact that the linear-prediction FIR filter of Fig. 9 has a "corresponding" IIR version in the sense described above raises the following question: What is the IIR filter structure that "corresponds" to the FIR structure of Fig. 3 ? The answer is easy to find, but the resulting structures do not seem to have particularly interesting properties. For example, the denominator of the IIR version turns out to be $P_{N-1}(z)$, which is an arbitrary (not necessarily minimum-phase) polynomial. Thus, even with all $k_{m}^{2}<1$, there is no guarantee that the IIR structure results in stable
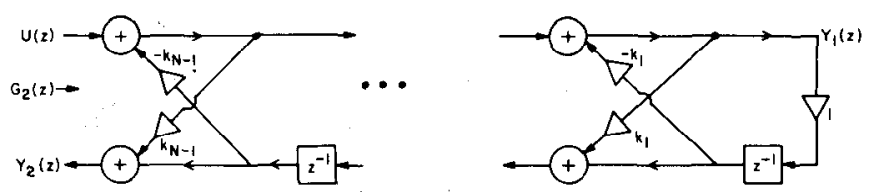

Fig. 10. The IIR countcrpart of the lincar-prediction FIR lattice of Fig. 9. Here, $G_{2}(z)=Y_{2}(z) / U(z)$ and $G_{1}(z)=Y_{1}(z) / U(z)$.

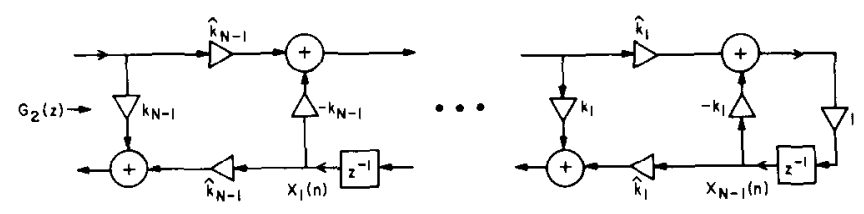

Fig. 11. The normalized version of Fig. 10.

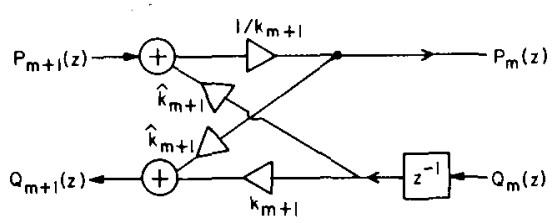

Fig. 12. The basic lattice section of the "corresponding" IIR filter.

transfer functions under a quantized environment. For completeness of discussion, Fig. 12 shows the basic building block of this IIR filter. The transfer matrix of this building block is not LBR, and, hence, the IIR structure of Fig. 12 has no relation to orthogonal implementations.

\section{Relation to Continuous-Time Electrical Networks}

Since each building block in our structure of Fig. 3 is lossless, it is possible, in principle, to find a counterpart of our structures in the continuous-time domain. This can be done by employing the bilinear transformation and the wave-to-immittance transformations. Subsequent to the revision of this manuscript, a recent reference [32] was brought to our attention by its author, in a private conversation. This reference indicates how a FIR filter can be derived from certain electrical networks by the wave-filter approach.

\section{Extension of the Synthesis Procedure to $M$-COMPONENT FIR ALLPASS FUNCTIONS}

The ideas of Section III can be extended in order to synthesize a set of $M$ FIR transfer functions $P_{N-1}(z)$, $Q_{N-1}(z), \cdots, S_{N-1}(z)$ such that

$$
\left|P_{N-1}\left(e^{j \omega}\right)\right|^{2}+\left|Q_{N-1}\left(e^{j \omega}\right)\right|^{2}+\cdots+\left|S_{N-1}\left(e^{j \omega}\right)\right|^{2}=1 .
$$

A typical application of such filter banks is in subband coding, and Sections VIII and IX deal with some of the applications. The purpose of this section is to outline a procedure for synthesizing a structure for such filter banks, based on a cascade of lossless lattice building blocks. We begin by considering three-component FIR allpass vectors.

In order to synthesize a three-component FIR allpass function of the form $G_{N-1}(z)=\left[P_{N-1}(z)\right.$ $\left.Q_{N-1}(z) R_{N-1}(z)\right]^{t}$ as a cascade of appropriate lattice building blocks, we should first extend the "order-reduction" scheme of Section III to the three-component case. 


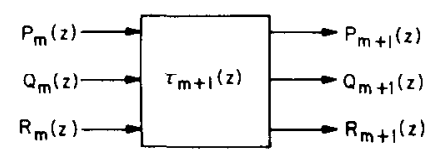

Fig. 13. Realization of $\boldsymbol{G}_{m+1}(z)$.

Accordingly, let us consider a three-component FIR allpass function

$$
\begin{array}{r}
G_{m+1}(z)=\left[\begin{array}{lll}
P_{m+1}(z) & Q_{m+1}(z) & R_{m+1}(z)
\end{array}\right]^{t} \\
\tilde{P}_{m+1}(z) P_{m+1}(z)+\tilde{Q}_{m+1}(z) Q_{m+1}(z) \\
+\tilde{R}_{m+1}(z) R_{m+1}(z)=1
\end{array}
$$

with

$$
\begin{aligned}
P_{m+1}(z) & =\sum_{n=0}^{m+1} p_{m+1, n} z^{-n} \\
Q_{m+1}(z) & =\sum_{n=0}^{m+1} q_{m+1, n} z^{-n} \\
R_{m+1}(z) & =\sum_{n=0}^{m+1} r_{m+1, n} z^{-n}
\end{aligned}
$$

where at least one of $p_{m+1, m+1}, q_{m+1, m+1}, r_{m+1, m+1}$ is nonzero. Let us assume $p_{m+1, m+1} \neq 0$, without loss of generality. We wish to realize the given function $G_{m+1}(z)$ starting from an appropriately defined lower order FIR allpass function

$$
\boldsymbol{G}_{m}(z)=\left[\begin{array}{lll}
P_{m}(z) & Q_{m}(z) & R_{m}(z)
\end{array}\right]^{t}
$$

by means of the interconnection of Fig. 13, where $\mathscr{T}_{m+1}(z)$ is an LBR transfer matrix. Indeed, this turns out to be possible, as demonstrated by the following procedure. First, define

where

$$
P_{m}(z)=k_{1} P_{m+1}(z)+\hat{k}_{1} Q_{m+1}(z)
$$

$$
\begin{aligned}
& k_{1}=\frac{-q_{m+1, m+1}}{\sqrt{p_{m+1, m+1}^{2}+q_{m+1, m+1}^{2}}} \\
& \hat{k}_{1}=\frac{p_{m+1, m+1}}{\sqrt{p_{m+1, m+1}^{2}+q_{m+1, m+1}^{2}}} .
\end{aligned}
$$

Clearly, the highest term in the right-hand side of (42) cancels, and moreover $k_{1}^{2} \leqslant 1$. Next, define $Q_{m}(z)$ by

${ }^{z}{ }^{1} Q_{m}(z)=k_{2}\left[\hat{k}_{1} P_{m+1}(z)-k_{1} Q_{m+1}(z)\right]+\hat{k}_{2} R_{m+1}(z)$.

The polynomial $Q_{m}(z)$ represents a causal FIR transfer function of order $m$ if and only if

$$
k_{2}\left[\hat{k}_{1} p_{m+1,0}-k_{1} q_{m+1,0}\right]+\hat{k}_{2} r_{m+1,0}=0 .
$$

But the allpass property given by (39b) implies, in particular,

$$
p_{m+1,0} p_{m+1, m+1}+q_{m+1,0} q_{m+1, m+1}+r_{m+1,0} r_{m+1, m+1}=0 .
$$

In view of (43) and (46), eq. (45) becomes

$$
-k_{2} \frac{r_{m+1, m+1} \cdot r_{m+1,0}}{\sqrt{p_{m+1, m+1}^{2}+q_{m+1, m+1}^{2}}}+\hat{k}_{2} r_{m+1,0}=0 .
$$

Inspection of (47) reveals that the following choices of $k_{2}$ and $\hat{k}_{2}$ are appropriate:

$$
\begin{aligned}
& k_{2}=\frac{\sqrt{p_{m+1, m+1}^{2}+q_{m+1, m+1}^{2}}}{\sqrt{p_{m+1, m+1}^{2}+q_{m+1, m+1}^{2}+r_{m+1, m+1}^{2}}} \\
& \hat{k}_{2}=\frac{r_{m+1, m+1}}{\sqrt{p_{m+1, m+1}^{2}+q_{m+1, m+1}^{2}+r_{m+1, m+1}^{2}}} .
\end{aligned}
$$

We have thus produced two lower order polynomials $P_{m}(z), Q_{m}(z)$ by employing the following equations:

$$
\left[\begin{array}{c}
P_{m}(z) \\
z^{-1} Q_{m}(z)
\end{array}\right]=\left[\begin{array}{ccc}
k_{1} & \hat{k}_{1} & 0 \\
k_{2} \hat{k}_{1} & -k_{2} k_{1} & \hat{k}_{2}
\end{array}\right]\left[\begin{array}{c}
P_{m+1}(z) \\
Q_{m+1}(z) \\
R_{m+1}(z)
\end{array}\right]
$$

where $k_{1}^{2}, k_{2}^{2}<1$, and $k_{i}^{2}+\hat{k}_{i}^{2}=1, i=1,2$. We finally wish to produce the third $m$ th-order polynomial $R_{m}(z)$ such that $\left[\begin{array}{lll}P_{m}(z) & z^{-1} Q_{m}(z) & R_{m}(z)\end{array}\right]^{t}$ (or equivalently $\left.\left[P_{m}(z) Q_{m}(z) R_{m}(z)\right]^{t}\right)$ is FIR allpass. This can be accomplished by "completing" the $2 \times 3$ matrix in (50) by adding a third row such that the $3 \times 3$ matrix becomes orthogonal

$$
\begin{aligned}
{\left[\begin{array}{c}
P_{m}(z) \\
z^{-1} Q_{m}(z) \\
R_{m}(z)
\end{array}\right] } & =\left[\begin{array}{ccc}
k_{1} & \hat{k}_{1} & 0 \\
k_{2} \hat{k}_{1} & -k_{2} k_{1} & \hat{k}_{2} \\
\hat{k}_{2} \hat{k}_{1} & -\hat{k}_{2} k_{1} & -k_{2}
\end{array}\right]\left[\begin{array}{l}
P_{m+1}(z) \\
Q_{m+1}(z) \\
R_{m+1}(z)
\end{array}\right] \\
& =\mathscr{K}_{m+1}\left[\begin{array}{c}
P_{m+1}(z) \\
Q_{m+1}(z) \\
R_{m+1}(z)
\end{array}\right]
\end{aligned}
$$

In view of the fact that $k_{i}^{2}+\hat{k}_{i}^{2}=1, i=1,2$, the matrix $\mathscr{K}_{m+1}$ is indeed orthogonal, hence

$$
\begin{aligned}
\tilde{P}_{m}(z) & P_{m}(z)+\tilde{Q}_{m}(z) Q_{m}(z)+\tilde{R}_{m}(z) R_{m}(z) \\
= & \tilde{P}_{m+1}(z) P_{m+1}(z)+\tilde{Q}_{m+1}(z) Q_{m+1}(z) \\
& +\tilde{R}_{m+1}(z) R_{m+1}(z)=1 .
\end{aligned}
$$

It only remains to show that $R_{m}(z)$ is of order $m$. Now, the highest coefficient of $R_{m}(z)$ is given, according to (51), by

$$
r_{m, m+1}=\hat{k}_{2}\left(\hat{k}_{1} p_{m+1, m+1}-k_{1} q_{m+1, m+1}\right)-k_{2} r_{m+1, m+1} .
$$

Substituting from (43), (48), and (49), eq. (53) immediately reveals that $r_{m, m+1}=0$. Thus, $R_{m}(z)$ is an $m$ th-order polynomial. In conclusion, we have generated a lower order FIR allpass vector $G_{m}(z)$ as in (41) starting from the FIR allpass vector $\boldsymbol{G}_{m+1}(z)$ of (39a), such that (51) holds. 


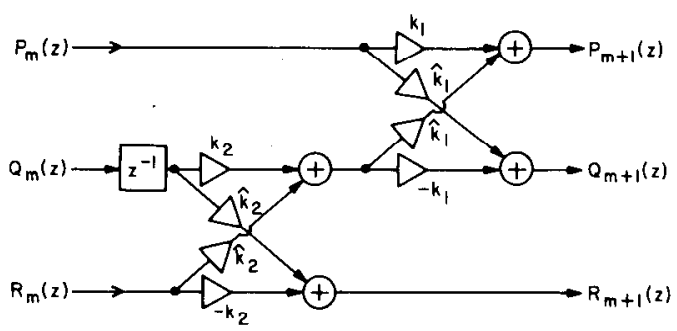

Fig. 14. Complete implementation of $\boldsymbol{G}_{m+1}(z)$ in terms of $\boldsymbol{G}_{m}(z)$.

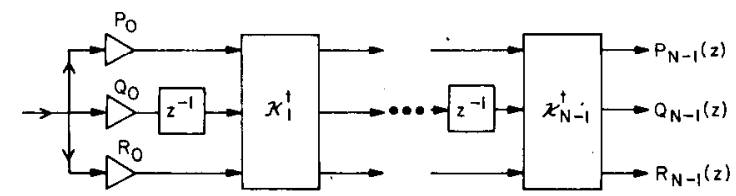

Fig. 15. Overall cascaded-lattice implementation of $G_{N-1}(z)$.

Inversion of (51) leads to

$$
\begin{aligned}
{\left[\begin{array}{c}
P_{m+1}(z) \\
Q_{m+1}(z) \\
R_{m+1}(z)
\end{array}\right] } & =\left[\begin{array}{ccc}
k_{1} & \hat{k}_{1} k_{2} & \hat{k}_{1} \hat{k}_{2} \\
\hat{k}_{1} & -k_{1} k_{2} & -k_{1} \hat{k}_{2} \\
0 & \hat{k}_{2} & -k_{2}
\end{array}\right]\left[\begin{array}{c}
P_{m} \\
z^{-1} Q_{m} \\
R_{m}
\end{array}\right] \\
& =\mathscr{K}_{m+1}^{t}\left[\begin{array}{c}
P_{m} \\
z^{-1} Q_{m} \\
R_{m}
\end{array}\right]
\end{aligned}
$$

Equivalently

$$
\left[\begin{array}{l}
P_{m+1}(z) \\
Q_{m+1}(z) \\
R_{m+1}(z)
\end{array}\right]-\mathscr{T}_{m+1}(z)\left[\begin{array}{l}
P_{m} \\
Q_{m} \\
R_{m}
\end{array}\right]
$$

where the transfer matrix

$$
\mathscr{T}_{m+1}(z)=\left[\begin{array}{ccc}
k_{1} & z^{-1} \hat{k}_{1} k_{2} & \hat{k}_{1} \hat{k}_{2} \\
\hat{k}_{1} & -z^{-1} k_{1} k_{2} & -k_{1} \hat{k}_{2} \\
0 & z^{-1} \hat{k}_{2} & -k_{2}
\end{array}\right]
$$

is clearly LBR. Notice that the orthogonal matrix $\mathscr{K}_{m+1}^{t}$ can be factorized into two planar rotations

$$
\mathscr{K}_{m+1}^{t}=\left[\begin{array}{ccc}
k_{1} & \hat{k}_{1} & 0 \\
\hat{k}_{1} & -k_{1} & 0 \\
0 & 0 & 1
\end{array}\right]\left[\begin{array}{ccc}
1 & 0 & 0 \\
0 & k_{2} & \hat{k}_{2} \\
0 & \hat{k}_{2} & -k_{2}
\end{array}\right] .
$$

Fig. 14 shows the corresponding implementation of (54). Notice that the quantities $k_{1}$ and $k_{2}$ in (57) should have had an additional subscript $(m+1)$ in order to indicate the "section number." As a matter of notational simplicity, we omit this subscript. It is clear that by repeated application of the order-reduction procedure, we can synthesize any three-component FIR allpass vector in the form of a normalized cascaded-lattice structure (or orthogonal structure) as shown in Fig. 15, where constants $P_{0}, Q_{0}, R_{0}$ are such that $P_{0}^{2}+Q_{0}^{2}+R_{0}^{2}=1$.

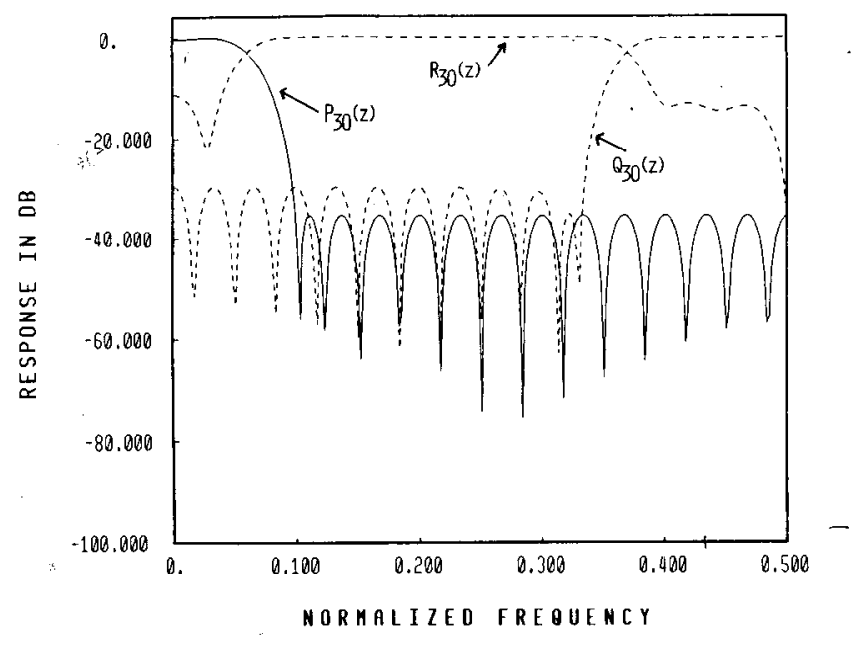

(a)

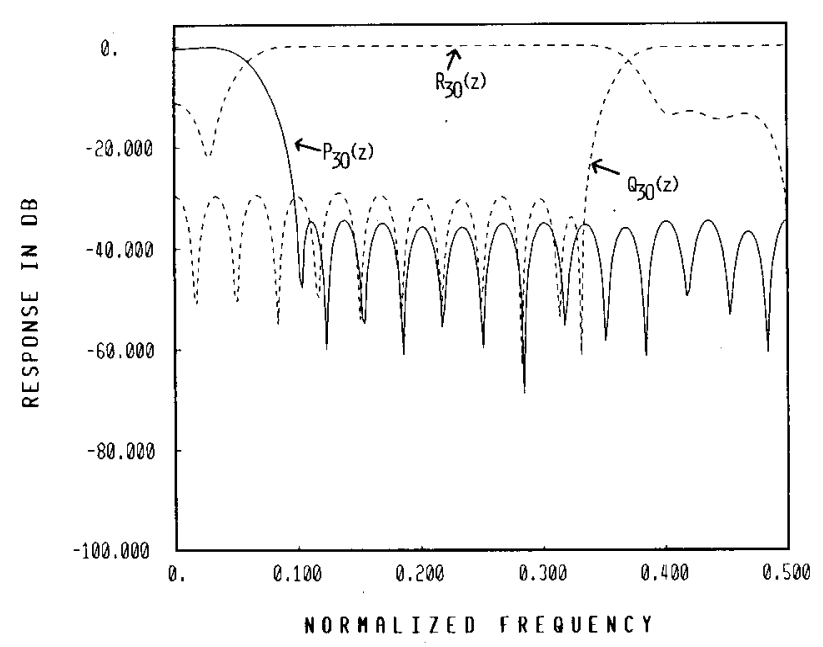

(b)

Fig. 16. (a) Example 2. The frequency responses for unquantized LBR lattice. (b) Example 2. The frequency responses for 4-bit quantized LBR lattice.

\section{A. Design Example 2}

Let us consider the transfer function $G_{30}(z)=\left[P_{30}(z)\right.$ $\left.Q_{30}(z) \quad R_{30}(z)\right]^{t}$, where $P_{30}(z), Q_{30}(z), R_{30}(z)$ are obtained as follows: $P_{30}(z)$ is a 30 th-order linear-phase equiripple FIR low-pass transfer function with band edges $\omega_{p}=0.04(2 \pi)$ radians and $\omega_{s}=(0.10) 2 \pi$ radians, designed by invoking the McClellan-Parks program [27]. $Q_{30}(z)$ is a 30th-order linear-phase FIR bandpass transfer function, generated by modulating the impulse response of $P_{30}(z)$, i.e.,

$$
q_{30, n}=2 p_{30, n} \cos \omega_{0}\left(n-\frac{N-1}{2}\right)
$$

where $\omega_{0}=(0.43) 2 \pi$, and $N-1=30$. The transfer functions $P_{30}(z)$ and $Q_{30}(z)$ are scaled so that $\left|P_{30}\left(e^{j \omega}\right)\right|^{2}+$ $\left|Q_{30}\left(e^{j \omega}\right)\right|^{2} \leqslant 1$. The third FIR function $R_{30}(z)$ is chosen such that ( $39 \mathrm{~b})$ holds. The required spectral factorization is done using [26]. The frequency response of the resulting cascaded lossless lattice structure is shown in Fig. 16(a). 
The quantized lattice implementation, with 4 bits per $k$-parameter, has a frequency response as shown in Fig. 16(b), and is close to the ideal response of Fig. 16(a).

\section{B. Further Generalizations}

Recall the order-reduction scheme of Section III. Given the polynomials $P_{m+1}(z)$ and $Q_{m+1}(z)$, we first selected $k_{m+1}$ so that $P_{m}(z)$ defined by (12) has order $m$. We found that, if $Q_{m}(z)$ is then defined as in (17), it automatically has degree $m$. Similarly earlier in this section, we found $k_{1}$ and $k_{2}$ such that $P_{m}(z)$ and $Q_{m}(z)$ have order $m$. Then the definition of $R_{m}(z)$ according to (51) automatically ensures that $R_{m}(z)$ has the reduced order $m$.

The above facts give us evidence that there is something fundamental about allpass vectors and LBR transfer matrices which forces this order-reduction automatically on the $M$ th component, provided the $k$-parameters are chosen to ensure order-reduction of the first $M-1$ compo- such that

$$
Q_{m}(z)=k_{2}\left[\hat{k}_{1} P_{m+1}(z)-k_{1} Q_{m+1}(z)\right]+\hat{k}_{2} R_{m+1}(z)
$$

is of order $m$, i.e., has coefficient of $z^{-(m+1)}$ equal to zero. Moreover, $k_{2}$ and $\hat{k}_{2}$ are such that $k_{2}^{2} \leqslant 1$ and $k_{2}^{2}+\hat{k}_{2}^{2}=1$ (see (61)). Once we form $Q_{m}(z)$, we can next define an $(m+1)$ th-degree polynomial

$$
S(z)=\hat{k}_{2}\left[\hat{k}_{1} P_{m+1}(z)-k_{1} Q_{m+1}(z)\right]-k_{2} R_{m+1}(z)
$$

and then proceed to generate the $m$ th-degree polynomial

$$
R_{m}(z)=k_{3} S(z)+\hat{k}_{3} V_{m+1}(z)
$$

and so on. In this manner, given the $M$-component FIR vector $\boldsymbol{G}_{m+1}(z)=\left[P_{m+1}(z), Q_{m+1}(z), R_{m+1}(z), \cdots\right.$, $\left.U_{m+1}(z), S_{m+1}(z)\right]^{t}$, we can always generate the $M-1$ polynomials of order $m,\left\{P_{m}(z), Q_{m}(z), R_{m}(z), \cdots\right.$, $\left.U_{m}(z)\right\}$. Thus

$$
\left[\begin{array}{c}
P_{m}(z) \\
Q_{m}(z) \\
R_{m}(z) \\
\vdots \\
U_{m}(z)
\end{array}\right]=\left[\begin{array}{ccccccc}
k_{1} & \hat{k}_{1} & 0 & 0 & 0 & \ldots & 0 \\
k_{2} \hat{k}_{1} & -k_{2} k_{1} & \hat{k}_{2} & 0 & 0 & \ldots & 0 \\
k_{3} \hat{k}_{2} \hat{k}_{1} & -k_{3} \hat{k}_{2} k_{1} & -k_{3} k_{2} & \hat{k}_{3} & 0 & \ldots & 0 \\
\vdots & & & \ddots & & & \\
& & & & \ddots & & \\
& & & & & \ddots & \hat{k}_{M-1}
\end{array}\right]\left[\begin{array}{c}
P_{m+1}(z) \\
Q_{m+1}(z) \\
R_{m+1}(z) \\
\vdots \\
U_{m+1}(z) \\
S_{m+1}(z)
\end{array}\right]
$$

nents. We now proceed to establish a certain property which explains the reason why we can always force an order-reduction in the above manner.

First notice that, given two arbitrary polynomials $P_{L}(z)$ and $Q_{L}(z)$ (not necessarily forming an allpass vector)

$$
\begin{aligned}
& P_{L}(z)=\sum_{n=0}^{L} p_{n} z^{-n} \\
& Q_{L}(z)=\sum_{n=0}^{L} q_{n} z^{-n}
\end{aligned}
$$

with $p_{L} \neq 0$, we can always find a lower order polynomial $P_{L-1}(z)$ simply by defining

$$
P_{L-1}(z)=k P_{L}(z)+\hat{k} Q_{L}(z)
$$

where

$$
\begin{aligned}
& k=-q_{L} / \sqrt{p_{L}^{2}+q_{L}^{2}} \\
& \hat{k}=p_{L} / \sqrt{p_{L}^{2}+q_{L}^{2}} .
\end{aligned}
$$

Clearly, $k^{2}<1$ and $k^{2}+\hat{k}^{2}=1$.

Now, assume that we have a set of $M$ polynomials of order $\leqslant m+1\left\{P_{m+1}(z), Q_{m+1}(z), R_{m+1}(z)\right.$, $\left.V_{m+1}(z), \cdots, U_{m+1}(z), S_{m+1}(z)\right\}$, with at least one polynomial having order $m+1$. Thus, let $p_{m+1, m+1} \neq 0$. We can always generate an $m$ th-order polynomial $P_{m}(z)$ as in (42) simply by choosing $k_{1}$ and $\hat{k}_{1}$ as in (43). Once this is done, the quantity $\left[\hat{k}_{1} P_{m+1}(z)-k_{1} Q_{m+1}(z)\right]$ is just another $(m+1)$ th-order polynomial, and we can always find $k_{2}, \hat{k}_{2}$
In (65), each $k_{i}$ satisfies $k_{i}^{2} \leqslant 1$ and $k_{i}^{2}+\hat{k}_{i}^{2}=1$. Let us now define a polynomial $J_{m}(z)$ simply by appending a row to the $(M-1) \times M$ matrix of $(65)$ such that the resulting $M \times M$ matrix is orthogonal

$$
\begin{aligned}
& \boldsymbol{H}_{m}(z) \triangleq\left[P_{m}(z) Q_{m}(z) R_{m}(z) \cdots U_{m}(z) J_{m}(z)\right]^{t} \\
&=\mathscr{K}_{m+1} \boldsymbol{G}_{m+1}(z) \\
& \mathscr{K}_{m+1}^{t} \mathscr{K}_{m+1}=\boldsymbol{I}_{\boldsymbol{M}} .
\end{aligned}
$$

If we now impose the additional restriction that $\boldsymbol{G}_{m+1}(z)$ be (FIR) allpass, then $\boldsymbol{H}_{m}(z)$ is also (FIR) allpass because of (67). Under this condition, we claim that $J_{m}(z)$ is automatically of the form

$$
J_{m}(z)=J_{m, 1} z^{-1}+J_{m, 2} z^{-2}+\cdots+J_{m, m+1} z^{-(m+1)}
$$

i.e., $J_{m, 0}=0$. In other words, $z J_{m}(z)$ represents a causal, $m$ th-order FIR filter. In order to see this, it only remains to note that the allpass property of $\boldsymbol{H}_{m}(z)$, i.e.,

$$
\tilde{P}_{m}(z) P_{m}(z)+\tilde{Q}_{m}(z) Q_{m}(z)+\cdots+\tilde{J}_{m}(z) J_{m}(z)=1
$$

implies in particular that

$$
J_{m, 0} J_{m, m+1}=0
$$
have

$$
p_{m, m+1}=q_{m, m+1}=\cdots u_{m, m+1}=0 .
$$

If $J_{m, m+1}=0$ in (70), then all the components in $\boldsymbol{H}_{m}(z)$ because, by construction of $P_{m}(z), Q_{m}(z), \cdots, U_{m}(z)$, we 


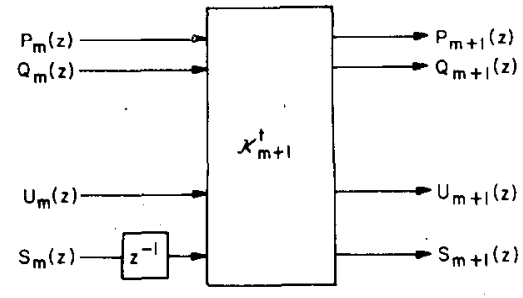

(a)

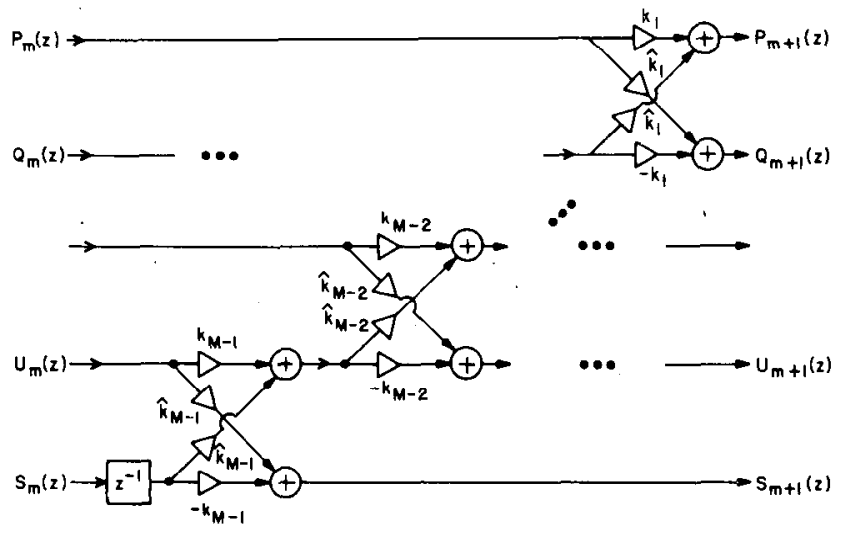

(b)

Fig. 17. (a) Implementation of $\boldsymbol{G}_{\boldsymbol{m}+1}(z)$ in terms of $\boldsymbol{G}_{m}(z)$. (b) Internal details of $\mathscr{K}_{m+1}^{m}$ in Fig. 17(a).

are $m$ th-order polynomials, and hence

$$
\boldsymbol{G}_{m+1}(z)=\mathscr{K}_{m+1}^{r t} \boldsymbol{H}_{m}(z)
$$

cannot have order $m+1$. Thus, (70) necessarily implies $J_{m, 0}=0$. This shows that $J_{m}(z)$ is of the form shown in (68). Having established that $J_{m}(z)$ is as in (68), we next define the allpass vector $G_{m}(z)$ to be

$$
\begin{aligned}
\boldsymbol{G}_{m}(z) & =\left[P_{m}(z) Q_{m}(z) \cdots U_{m}(z) S_{m}(z)\right]^{t} \\
S_{m}(z) & \triangleq z J_{m}(z) .
\end{aligned}
$$

We have thus shown how to construct an $m$ th-order FIR allpass vector $\boldsymbol{G}_{m}(z)$ from an $(m+1)$ th-order FIR allpass vector $\boldsymbol{G}_{m+1}(z)$. Fig. 17(a) shows how $\boldsymbol{G}_{m+1}(z)$ can be reconstructed from $\boldsymbol{G}_{m}(z)$. Notice that the circuit of Fig. 17(a) involves only one delay unit $z^{-1}$.

In order to complete the process, it remains to indicate how the orthogonal matrix $\mathscr{K}_{m+1}$ should be obtained. For this, let us consider an example where $M=4$; the $3 \times 4$ matrix of (65) is

$$
\mathscr{L}=\left[\begin{array}{cccc}
k_{1} & \hat{k}_{1} & 0 & 0 \\
k_{2} \hat{k}_{1} & -k_{2} k_{1} & \hat{k}_{2} & 0 \\
k_{3} \hat{k}_{2} \hat{k}_{1} & -k_{3} \hat{k}_{2} k_{1} & -k_{3} k_{2} & \hat{k}_{3}
\end{array}\right] .
$$

The $4 \times 4$ orthogonal matrix $\mathscr{K}_{m+1}$ is obtained by appending the row

$$
\left[\begin{array}{llll}
\hat{k}_{3} \hat{k}_{2} \hat{k}_{1} & -\hat{k}_{3} \hat{k}_{2} k_{1}-\hat{k}_{3} k_{2}-k_{3}
\end{array}\right]
$$

to (75). It is easily seen that $\mathscr{K}_{m+1}$ can be written as

$$
\begin{aligned}
\mathscr{K}_{m+1}= & {\left[\begin{array}{cccc}
1 & 0 & 0 & 0 \\
0 & 1 & 0 & 0 \\
0 & 0 & k_{3} & \hat{k}_{3} \\
0 & 0 & \hat{k}_{3} & -k_{3}
\end{array}\right]\left[\begin{array}{cccc}
1 & 0 & 0 & 0 \\
0 & k_{2} & \hat{k}_{2} & 0 \\
0 & \hat{k}_{2} & -k_{2} & 0 \\
0 & 0 & 0 & 1
\end{array}\right] } \\
& \cdot\left[\begin{array}{cccc}
k_{1} & \hat{k}_{1} & 0 & 0 \\
\hat{k}_{1} & -k_{1} & 0 & 0 \\
0 & 0 & 1 & 0 \\
0 & 0 & 0 & 1
\end{array}\right] .
\end{aligned}
$$

Each factor in (76) is an orthogonal matrix involving one planar rotation. Thus, the orthogonal matrix $\mathscr{K}_{m+1}$ involves a succession of three planar rotations. In general, for $M$-component FIR filter synthesis, the orthogonal matrix $\mathscr{K}_{m+1}$ in (66) can be written as

$$
\begin{aligned}
\mathscr{K}_{m+1}= & {\left[\begin{array}{ccc}
\boldsymbol{I} & \boldsymbol{O} & \boldsymbol{O} \\
\boldsymbol{O} & k_{M-1} & \hat{k}_{M-1} \\
\boldsymbol{O} & \hat{k}_{M-1} & -k_{M-1}
\end{array}\right] } \\
& \cdot\left[\begin{array}{cccc}
\boldsymbol{I} & \boldsymbol{O} & \boldsymbol{O} & \boldsymbol{O} \\
\boldsymbol{O} & k_{M-2} & \hat{k}_{M-2} & \boldsymbol{O} \\
\boldsymbol{O} & \hat{k}_{M-2} & -k_{M-2} & \boldsymbol{O} \\
\boldsymbol{O} & \boldsymbol{O} & \boldsymbol{O} & \boldsymbol{I}
\end{array}\right] \ldots \\
& \cdot\left[\begin{array}{ccc}
k_{1} & \hat{k}_{1} & \boldsymbol{O} \\
\hat{k}_{1} & -k_{1} & \boldsymbol{O} \\
\boldsymbol{O} & \boldsymbol{O} & \boldsymbol{I}
\end{array}\right]
\end{aligned}
$$

where $\boldsymbol{O}$ and $\boldsymbol{I}$ in (77) stand for the identity matrix and null matrices of appropriate dimensions. Accordingly, (72) gives

$$
\begin{aligned}
\boldsymbol{G}_{m+1}(z)= & {\left[\begin{array}{c}
P_{m+1}(z) \\
Q_{m+1}(z) \\
R_{m+1}(z) \\
\vdots \\
S_{m+1}(z)
\end{array}\right]=\left[\begin{array}{ccc}
k_{1} & \hat{k}_{1} & \boldsymbol{O} \\
\hat{k}_{1} & -k_{1} & \boldsymbol{O} \\
\boldsymbol{O} & \boldsymbol{O} & \boldsymbol{I}
\end{array}\right] \ldots } \\
& \cdot\left[\begin{array}{ccc}
\boldsymbol{I} & \boldsymbol{O} & \boldsymbol{O} \\
\boldsymbol{O} & k_{M-1} & \hat{k}_{M-1} \\
\boldsymbol{O} & \hat{k}_{M-1} & -k_{M-1}
\end{array}\right]\left[\begin{array}{c}
P_{m}(z) \\
Q_{m}(z) \\
R_{m}(z) \\
\vdots \\
z^{-1} S_{m}(z)
\end{array}\right] .
\end{aligned}
$$

Thus, given the allpass vector $G_{m}(z)=\left[P_{m}(z) \cdots S_{m}(z)\right]^{t}$, we can reconstruct $G_{m+1}(z)$ as shown in Fig. 17(b), and this involves one delay operator and $M-1$ planar rotation operators. Clearly, an $M$-component FIR allpass vector of order $N-1$ can be implemented by cascading $N-1$ building blocks, as shown in Fig. 18, where each $\mathscr{T}_{m+1}(z)$ is an 


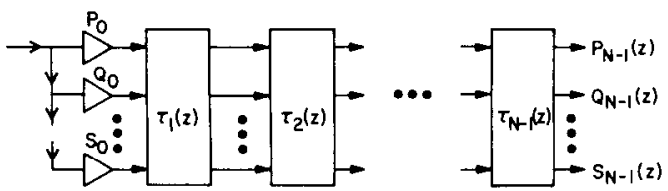

Fig. 18. Cascade of $N-1$ building blocks, where each $\mathscr{T}_{k}(z)$ has the form in Fig. 17.

$M \times M$ LBR transfer matrix of the form

$$
\begin{aligned}
\mathscr{T}_{m+1}(z)= & {\left[\begin{array}{ccc}
k_{1} & \hat{k}_{1} & \boldsymbol{O} \\
\hat{k}_{1} & -k_{1} & \boldsymbol{O} \\
\boldsymbol{O} & \boldsymbol{O} & \boldsymbol{I}
\end{array}\right] \ldots } \\
& \cdot\left[\begin{array}{ccc}
\boldsymbol{I} & \boldsymbol{O} & \boldsymbol{O} \\
\boldsymbol{O} & k_{M-1} & z^{-1} \hat{k}_{M-1} \\
\boldsymbol{O} & \hat{k}_{M-1} & -z^{-1} k_{M-1}
\end{array}\right] .
\end{aligned}
$$

This leads to the cascaded normalized lattice structure or orthogonal implementation of the FIR allpass function $\boldsymbol{G}_{N-1}(z)=\left[\begin{array}{ll}P_{N-1}(z) & Q_{N-1}(z) \cdots S_{N-1}(z)\end{array}\right]^{t}$.

The $M$-input $M$-output building block for the cascaded FIR lattice structure has striking resemblance to a wellknown single-input single-output IIR digital filter structure, namely, the normalized Gray-and-Markel lattice structure [6] reproduced in Fig. 11 earlier. It can be shown [28] that, if the IIR single-input single-output allpass filter $G_{2}(z)$ in Fig. 11 is represented in state-variable form

$$
\begin{aligned}
\boldsymbol{x}(n+1) & =\boldsymbol{A} \boldsymbol{x}(n)+\boldsymbol{B} u(n) \\
\boldsymbol{y}(n) & =\boldsymbol{C} \boldsymbol{x}(n)+\boldsymbol{D} u(n)
\end{aligned}
$$

where $\boldsymbol{x}(n)=\left[x_{1}(n) \cdots x_{N-1}(n)\right]^{t}$, then the following $N \times N$ matrix:

$$
\mathscr{R}=\left[\begin{array}{ll}
D & C \\
B & A
\end{array}\right]
$$

is orthogonal, and moreover, is structurally similar to the $M \times M$ matrix $\mathscr{K}_{m+1}$ of (77). ${ }^{3}$

It should, however, be borne in mind that the matrix $\mathscr{R}$ in (82) pertains to a single-input single-output IIR allpass structure, whereas the matrix $\mathscr{K}_{m+i}$ of (77) pertains to one section of a one-input $M$-output FIR allpass function of arbitrary order $N-1$. Notice also that the IIR structure of Fig. 11 is related to the theory of orthogonal polynomials [6], [7], [11], whereas the FIR structures of Fig. 18 cannot be directly related to orthogonal polynomials. Finally, the IIR allpass cascaded lattice can in turn be related to the theory of linear prediction and Gram-Schmidt orthogonalization of random variables, whereas no such interpretation of the cascaded FIR lossless lattice structure of Fig. 18 seems to be evident.

\footnotetext{
${ }^{3}$ More precisely, if the quantity $N$ in Fig. 11 were the same as $M$, and if the subscripts on the $k$-parameters were read backwards, then $\mathscr{R}$ is equal to $\mathscr{K}_{m+1}$.
}

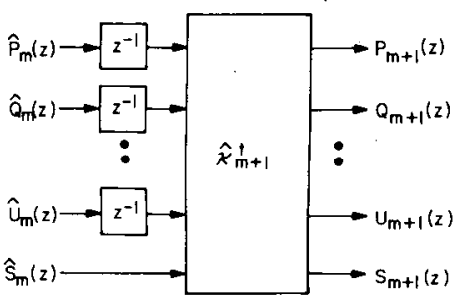

Fig. 19. Building blocks based on alternative order-reduction scheme.

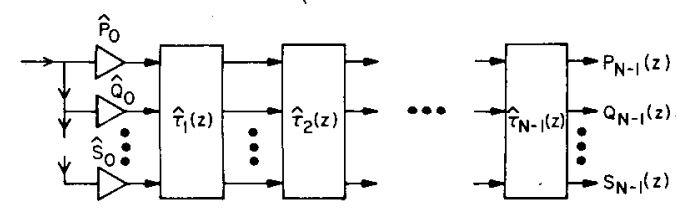

Fig. 20. Cascaded lattice corresponding to Fig. 19.

\section{Variations of the Cascaded-Lattice Implementation}

The procedure described above for the synthesis of an $M$-component FIR allpass vector does not necessarily lead to a unique structure. For example, the value of $k_{1}$ in (65) depends on which one of the $M$ polynomials is defined to be the first polynomial $P_{m+1}(z)$. Thus, at each one of the $N-1$ stages of iteration, we have a wide choice available. A permutation of the components in $\boldsymbol{G}_{m+1}(z)$ in general leads to more than a mere permutation in $G_{m}(z)$.

From the point of view of finite-wordlength behavior, there does not seem to be any reason to expect this multitude of equivalent structures to behave differently from each other. This is because all the structures are cascaded interconnections of orthogonal building blocks, with each building block being a succession of $M-1$ planar rotations. However, it might turn out that a particular realization has certain $k_{m}$ coefficients that have simple values (such as $k_{m}= \pm 1$ or 0 ). In general, it is difficult to anticipate this, and the choice of the "best structure" does not seem to be an easily tractable problem.

$\mathrm{We}$, however, wish to outline one particular variation of the synthesis procedure that we shall find useful in the analysis-synthesis filter-bank application of Section VIII. Recall that, when we constructed the lower-order function $\boldsymbol{G}_{m}(z)$ from $\boldsymbol{G}_{m+1}(z)$, we ensured that each one of the polynomials $P_{m}(z), Q_{m}(z), \cdots, U_{m}(z)$ has the highest power (corresponding to $z^{-(m+1)}$ canceled, whereas $J_{m}(z)$ has the lowest power (constant term) canceled. Accordingly, Fig. 17(a), which reconstructs $\boldsymbol{G}_{m+1}(z)$ from $\boldsymbol{G}_{m}(z)$ involves only one delay operator.

Instead of proceeding as above, let us assume that we cancel the lowest power (constant term) in each one of $P_{m}(z), Q_{m}(z), \cdots, U_{m}(z)$, and then cancel the highest term (coefficient of $z^{-(m+1)}$ ) in $J_{m}(z)$. Then the resulting cascaded-lattice structure has building blocks as shown in Fig. 19, whereas the overall cascaded structure is as in Fig. 20. Notice that, for a given $\boldsymbol{G}_{m+1}(z)$, the set of polynomials $\left[\hat{P}_{m}(z) \cdots \hat{U}_{m}(z), \hat{S}_{m}(z)\right]$ in Fig. 19, and the orthogonal matrix $\mathscr{K}_{m+1}$ are in general different from the quantities $\left[P_{m}(z) \cdots U_{m}(z) S_{m}(z)\right]$ and $\mathscr{K}_{m+1}$ appearing in Fig. 17. 
Accordingly, we have two equivalent realizations, described by

$$
\begin{aligned}
\boldsymbol{G}_{N-1}(z)= & {\left[\begin{array}{c}
P_{N-1}(z) \\
Q_{N-1}(z) \\
\vdots \\
S_{N-1}(z)
\end{array}\right] } \\
= & \mathscr{T}_{N-1}(z) \mathscr{T}_{N-2}(z) \cdots \mathscr{T}_{1}(z)\left[\begin{array}{c}
P_{0} \\
Q_{0} \\
\vdots \\
S_{0}
\end{array}\right] \\
= & \mathscr{T}(z)\left[\begin{array}{c}
P_{0} \\
Q_{0} \\
\vdots \\
S_{0}
\end{array}\right]
\end{aligned}
$$

for Fig. 18 and

$$
G_{N-1}(z)=\hat{\mathscr{T}}_{N-1}(z) \cdots \hat{\mathscr{T}}_{1}(z)\left[\begin{array}{c}
\hat{P}_{0} \\
\hat{Q}_{0} \\
\vdots \\
\hat{S}_{0}
\end{array}\right]=\hat{\mathscr{T}}(z)\left[\begin{array}{c}
\hat{P}_{0} \\
\hat{Q}_{0} \\
\vdots \\
\hat{S}_{0}
\end{array}\right]
$$

for Fig. 20. Note that $\hat{\mathscr{T}}_{m}(z), \hat{\mathscr{T}}(z), \mathscr{T}_{m}(z)$, and $\mathscr{T}(z)$ are all $M \times M$ LBR transfer matrices. Moreover, the following property is satisfied by the constants involved:

$$
P_{0}^{2}+Q_{0}^{2}+\cdots+S_{0}^{2}=1, \quad \hat{P}_{0}^{2}+\hat{Q}_{0}^{2}+\cdots+\hat{S}_{0}^{2}=1 .
$$

Notice that Fig. 19 involves $M-1$ delays, and, accordingly, the implementation of Fig. 20 involves $(M-1)$ $(N-1)$ delays and is highly nonminimal in $z^{-1}$. In contrast, the structure of Fig. 18 requires a total of only $N-1$ delays (i.e., the smallest possible number for order $N-1$ ). In spite of this, as we shall elaborate on in Section VIII, the circuit of Fig. 20 actually enables us to generate another transposed structure with the smallest number $(N-1)$ of delays. This finds application in the analysis-synthesis bank of Section VIII.

Before we conclude this section, it should be noticed that, given an $M$-component FIR allpass vector $G_{N-1}(z)$, the procedure for synthesizing it as a cascade of the form shown in Fig. 18 involves matrix-polynomial manipulations, at each stage of order-reduction. Accordingly, there can be numerical inaccuracies of significant amount when $N$ and $M$ are large. While computerizing the synthesis procedure, double-precision arithmetic is recommended strongly.

\section{Finite-Wordlength Properties of the Cascaded-Lattice FIR Structures}

In view of the LBR (or orthogonality) property of the building blocks of Fig. 17, the cascade of Fig. 18 exhibits a number of interesting properties relating to finite-word-

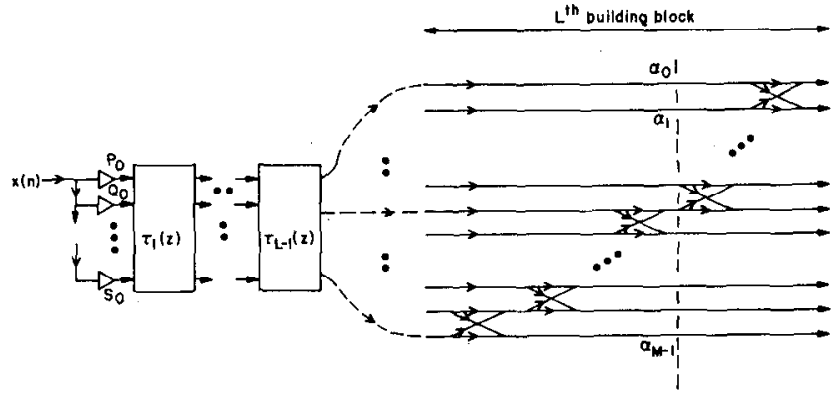

Fig. 21. Pertaining to internal-signal scaling.

length behavior. First notice that, since the $k_{l}$ parameters satisfy $k_{l}^{2} \leqslant 1$, they can be represented as fixed-point numbers in the range $[-1,1]$. We now proceed to look at a few other significant properties.

\section{A. Automatic Scaling of Internal Signals}

In order to scale a fixed-point implementation (with 2's complement arithmetic), it is sufficient to ensure that the signals which are input to internal multipliers be in the range $(-1,1)$. If the transfer function $H_{k}(z)$ from the filter input to the $k$ th multiplier input is such that its $L_{2}$ norm satisfies $\left\|H_{k}\left(e^{j \omega}\right)\right\|_{2} \leqslant 1$, and if this holds for all multipliers, then the structure is scaled in an $L_{2}$ sense.

Let us consider the cascade of Fig. 18. The transfer function (vector) from the filter input $x(n)$ to the set of $M$ internal points $\alpha_{0}, \alpha_{1}, \cdots, \alpha_{M-1}$ in the $L$ th building block (see Fig. 21) is given by

$$
\begin{aligned}
{\left[\begin{array}{c}
F_{0}(z) \\
F_{1}(z) \\
\vdots \\
F_{M-1}(z)
\end{array}\right]=} & {\left[\begin{array}{cccc}
\boldsymbol{I} & \boldsymbol{O} & \boldsymbol{O} & \boldsymbol{O} \\
\boldsymbol{O} & k_{l} & \hat{k}_{l} & \boldsymbol{O} \\
\boldsymbol{O} & \hat{k}_{l} & -k_{l} & \boldsymbol{O} \\
\boldsymbol{O} & \boldsymbol{O} & \boldsymbol{O} & \boldsymbol{I}
\end{array}\right] \ldots } \\
& \cdot\left[\begin{array}{ccc}
\boldsymbol{I} & \boldsymbol{O} & \boldsymbol{O} \\
\boldsymbol{O} & k_{M-1} & z^{-1} \hat{k}_{M-1} \\
\boldsymbol{O} & \hat{k}_{M-1} & -z^{-1} k_{M-1}
\end{array}\right] \\
& \cdot \mathscr{T}_{L-1}(z) \cdots \mathscr{T}_{1}(z)\left[\begin{array}{c}
P_{0} \\
Q_{0} \\
\vdots \\
S_{0}
\end{array}\right]
\end{aligned}
$$

Since $\mathscr{T}_{m}(z)$ are LBR and since $k_{m}^{2}+\hat{k}_{m}^{2}=1$, the entire $M \times M$ matrix on the right-hand side of (86) is LBR. Thus

$$
\begin{gathered}
\tilde{F}_{0}(z) F_{0}(z)+\cdots+\tilde{F}_{M-1}(z) F_{M-1}(z) \\
=P_{0}^{2}+Q_{0}^{2}+\cdots+S_{0}^{2}=1
\end{gathered}
$$

in view of (85). In other words

$$
\left|F_{0}\left(e^{j \omega}\right)\right|^{2}+\cdots+\left|F_{M-1}\left(e^{j \omega}\right)\right|^{2}=1, \quad \text { for all } \omega .
$$

Accordingly

$$
\left\|F_{k}\left(e^{j \omega}\right)\right\|_{2}^{2}=\int_{0}^{2 \pi}\left|F_{k}\left(e^{j \omega}\right)\right|^{2} \frac{d \omega}{2 \pi} \leqslant 1 .
$$




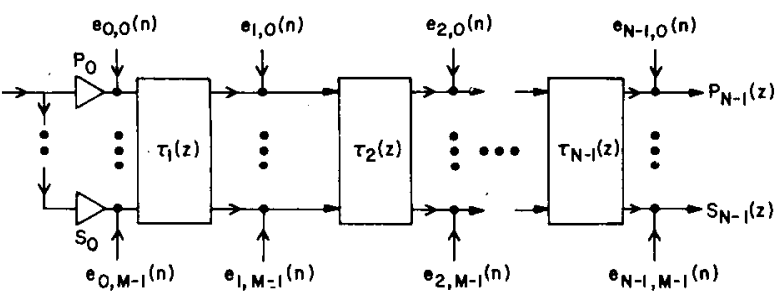

Fig. 22. Noise model for $M$-component cascaded lattice.

Thus, all the multiplier-inputs in Fig. 18 arc $L_{2}$-scaled, automatically.

\section{B. Roundoff-Noise Gain}

A noise model as shown in Fig. 22 can be assumed to hold for the structure of Fig. 18, provided that we do not permit any quantization inside the building block of Fig. 17. If this assumption is not true, then a slight modification of the following analysis can readily be made, so as to incorporate a general noise model.

The $M \times M$ transfer matrix from the $M$-component noise vector $\left[e_{m, 0}(n) \cdots e_{m, M-1}(n)\right]^{t}$ to the $M$-output terminals of the filter is given by

$$
\boldsymbol{S}(z) \triangleq \mathscr{T}_{N-1}(z) \cdots \mathscr{T}_{m+1}(z)
$$

which is clearly LBR. Assuming that each noise source is zero-mean and white with variance $\sigma_{e}^{2}$, and that different noise sources are mutually uncorrelated, we have for the total noise variance at the $k$ th-output of the filter due to noise sources at the input of $\mathscr{T}_{m+1}(z)$

$$
\sigma_{k}^{2}=\sigma_{e}^{2} \int_{0}^{2 \pi} \sum_{l=0}^{M-1}\left|S_{k l}\left(e^{j \omega}\right)\right|^{2} \frac{d \omega}{2 \pi}
$$

where $\boldsymbol{S}(z)=\left[\boldsymbol{S}_{k l}(z)\right]$. Since $\boldsymbol{S}(z)$ (and hence $\boldsymbol{S}^{t}(z)$ ) is LBR, (90) simplifies to

$$
\sigma_{k}^{2}=\sigma_{e}^{2} .
$$

Thus, the total noise variance at the $k$ th-output of the $M$-component filter, due to all noise sources, is

$$
\sigma_{k, \text { total }}^{2}=N \cdot \sigma_{e}^{2} \text {. }
$$

Recall that, for the direct-form implementation in an arbitrary (not necessarily linear-phase) FIR filter, the noise variance is given precisely by (92), provided that signals are quantized after each multiplication.

\section{A Word On Coefficient Sensitivity}

The structure of Fig. 18 is such that, as long as all the $k$-parameters satisfy $k_{l}^{2} \leqslant 1$, the function $G_{N-1}(z)$ is vector-allpass. Accordingly, the equality

$$
\left|P_{N-1}\left(e^{j \omega}\right)\right|^{2}+\left|Q_{N-1}\left(e^{j \omega}\right)\right|^{2}+\cdots+\left|S_{N-1}\left(e^{j \omega}\right)\right|^{2}=1
$$

holds for all $\omega$, regardless of the actual values of $k_{l}$. In other words, each component of $G_{N-1}(z)$ is structurally bounded, and according to the discussions in Section II, we expect low passband sensitivity.
However, it should be emphasized that, in practice, we cannot in general quantize both $k_{l}$ and $\hat{k}_{l}$ simultancously, and yet satisfy $k_{l}^{2}+\hat{k}_{l}^{2}=1$. Thus, under quantized conditions, (93) does not in general hold. A more accurate sensitivity analysis seems to be therefore appropriate. Let us assume that for each module of the form

$$
\left[\begin{array}{cc}
k_{l} & \hat{k}_{l} \\
\hat{k}_{l} & -k_{l}
\end{array}\right]
$$

the quantities $k_{l}$ and $\hat{k}_{l}$ are quantized independently. Under this condition, let us derive an expression for $\partial \boldsymbol{G}_{N-1}(z) / \partial k_{l}$. For simplicily, we consider only the structure of Fig. 3.

We have

$$
\begin{aligned}
\boldsymbol{G}_{N-1}(z) & =\left[\begin{array}{l}
P_{N-1}(z) \\
Q_{N-1}(z)
\end{array}\right] \\
& =\mathscr{T}_{N-1}(z) \mathscr{T}_{N-2}(z) \cdots \mathscr{T}_{1}(z)\left[\begin{array}{l}
P_{0} \\
Q_{0}
\end{array}\right]
\end{aligned}
$$

where the transfer matrices

$$
\mathscr{T}_{l}(z)=\left[\begin{array}{cc}
k_{l} & -\hat{k}_{l} z^{-1} \\
\hat{k}_{l} & k_{l} z^{-1}
\end{array}\right]
$$

are LBR and $P_{0}, Q_{0}$ satisfy $P_{0}^{2}+Q_{0}^{2}=1$. Let us denote

$$
\begin{aligned}
& \boldsymbol{S}_{l+1}(z) \triangleq \mathscr{T}_{N-1}(z) \cdots \mathscr{T}_{l+1}(z) \\
& \boldsymbol{R}_{l-1}(z) \triangleq \mathscr{T}_{l-1}(z) \cdots \mathscr{T}_{1}(z)
\end{aligned}
$$

whence

$$
\boldsymbol{G}_{N-1}(z)=\boldsymbol{S}_{l+1}(z) \mathscr{T}_{l}(z) \boldsymbol{R}_{l-1}(z) \cdot\left[\begin{array}{c}
P_{0} \\
Q_{0}
\end{array}\right]
$$

Now

$$
\begin{aligned}
\Delta(z) & \triangleq \frac{\partial \boldsymbol{G}_{N-1}(z)}{\partial k_{l}}=\left[\begin{array}{c}
\frac{\partial P_{N-1}(z)}{\partial k_{l}} \\
\frac{\partial Q_{N-1}(z)}{\partial k_{l}}
\end{array}\right] \\
& =\boldsymbol{S}_{l+1}(z)\left[\begin{array}{cc}
1 & 0 \\
0 & z^{-1}
\end{array}\right] \boldsymbol{R}_{l-1}(z)\left[\begin{array}{l}
P_{0} \\
Q_{0}
\end{array}\right]
\end{aligned}
$$

which shows that $\Delta(z)$ itself is LBR because $\boldsymbol{S}_{l+1}(z)$ and $\boldsymbol{R}_{t-1}(z)$ are LBR. Thus

$$
\left|\frac{\partial P_{N-1}\left(e^{j \omega}\right)}{\partial k_{l}}\right|^{2}+\left|\frac{\partial Q_{N-1}\left(e^{j \omega}\right)}{\partial k_{l}}\right|^{2}=1
$$

which shows that each variational term in (100) is bounded above by unity. This gives us additional insight concerning low-sensitivity behavior of the cascaded-lattice structure. The partial derivatives with respect to $\hat{k}_{l}$ also satisfy a relation similar to (100). 


\section{Applications in the Design of Digital FILTER BANKS}

The structure of Fig. 18 can also be used to implement a set of $M$ FIR transfer functions $\left[G_{0}(z) G_{1}(z) \cdots\right.$ $\left.G_{M-1}(z)\right]^{t}=G_{N-1}(z)$ such that they form a uniform filter bank [23]. Thus, we can let

$$
G_{k}(z)=G_{0}\left(z W^{k}\right), \quad k=0,1 \cdots M-1
$$

where $W \triangleq e^{-j 2 \pi / M}$ so that $G_{k}\left(e^{j \omega}\right)$ are shifted versions of $G_{0}\left(e^{j \omega}\right)$. If we have a set of $M$ FIR functions as in (101), and in addition if they are such that the vector $\boldsymbol{G}_{N-1}(z)$ is allpass, the structure of Fig. 18 can be further simplified, leading to a saving in computations by a factor of about $M$, for $N-1 \gg M$. In order to see this, note that

$$
G_{0}(z)=\dot{g}_{0}+g_{1} z^{-1}+\cdots+g_{N} z^{-(N-1)}
$$

can always be rewritten in the form [23], [29]

$$
G_{0}(z)=\sum_{k=0}^{M-1^{-}} z^{-k} E_{k}\left(z^{M}\right)
$$

where $E_{k}(z)=\left(g_{k}+g_{k+M^{2}} z^{-1}+g_{k+2 M^{2}} z^{-2}+\cdots\right)$. Equation (101) thus leads to

$$
\begin{aligned}
G_{k}(z)= & E_{0}\left(z^{M}\right)+W^{-k} z^{-1} E_{1}\left(z^{M}\right)+\cdots \\
& +W^{-k(M-1)} z^{-(M-1)} E_{M-1}\left(z^{M}\right) .
\end{aligned}
$$

In matrix form, we therefore have

$$
\boldsymbol{G}_{N-1}(z)=\boldsymbol{W}^{\dagger} \boldsymbol{E}(z)
$$

where $\boldsymbol{W}$ is the $M \times M$ DFT matrix (superscript dagger denotes transposed conjugation), and $\boldsymbol{E}(z)$ stands for

$\boldsymbol{E}(z)=\left[\begin{array}{llll}E_{0}\left(z^{M}\right) & z^{-1} E_{1}\left(z^{M}\right) \cdots & z^{-(M-1)} E_{M}{ }_{1}\left(z^{M}\right)\end{array}\right]^{t}$.

From (105) we have, in view of the allpass property of $\boldsymbol{G}_{N-1}(z)$

$1=\tilde{\boldsymbol{G}}_{N-1}(z) \boldsymbol{G}_{N-1}(z)=\tilde{\boldsymbol{E}}(z) \boldsymbol{W} \boldsymbol{W}^{\dagger} \boldsymbol{E}(z)=M \tilde{\boldsymbol{E}}(z) \boldsymbol{E}(z)$.

In other words, the vector $\sqrt{M} \boldsymbol{E}(z)$ also represents an FIR allpass vector. In fact, given an FIR vector $\boldsymbol{G}_{N-1}(z)$ with the components forming a uniform filter bank (i.e., (101) holding), $\boldsymbol{G}_{N-1}(z)$ is allpass if and only if $\sqrt{M} \boldsymbol{E}(z)$ is allpass. Note that, if the vector $\sqrt{M} \boldsymbol{E}(z)$ is allpass, then so is the vector

$$
\boldsymbol{F}(z)=\sqrt{M}\left[E_{0}(z) \quad E_{1}(z) \cdots E_{M-1}(z)\right]^{t} .
$$

Here, each function $E_{k}(z)$ has an order approximately equal to $(N-1) / M$. More specifically, letting

$$
N-1=m_{0}+m_{1} M, \quad 0 \leqslant m_{0}<M
$$

the polynomials $E_{0}(z), E_{1}(z), \cdots, E_{m_{0}}(z)$ have order $m_{1}$, whereas $E_{m_{0}+1}(z), \cdots, E_{M-1}(z)$ have order $\left(m_{1}-1\right)$. Since $\boldsymbol{F}(z)$ is an FIR allpass vector, it can be implemented as in Fig. 18 , as a cascade of only $m_{1}$ sections. We then replace each delay in the resulting structures with $z^{-M}$, and thus generate the set $\sqrt{M}\left[E_{0}\left(z^{M}\right), \cdots, E_{M}{ }_{1}\left(z^{M}\right)\right]^{t}$. The desired filter-bank vector $\boldsymbol{G}_{N-1}(z)$ is finally obtained by perform-

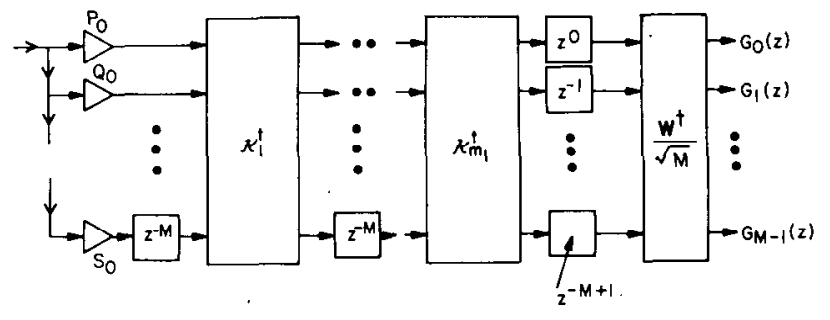

Fig. 23. Efficient implementation of uniform filter bank, using the cascaded lattice.

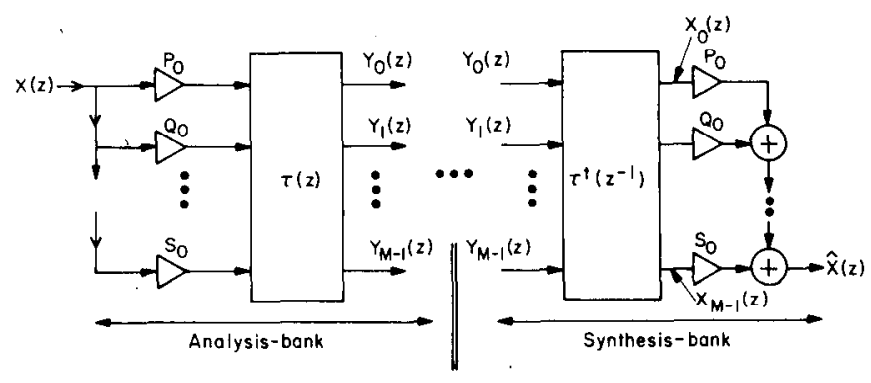

Fig. 24. The analysis-synthesis filter banks.

ing the $M$-point IDFT, as summarized in Fig. 23. Note that, as long as the IDFT operation $W^{\dagger}$ is performed using efficient techniques, the circuit of Fig. 23 is much more efficient than that of Fig. 18 for $N-1 \gg M$.

\section{A. Design of Analysis-Synthesis Banks}

Once again consider the filter bank of Fig. 18, implementing the vector $G_{N-1}(z)=\left[G_{0}(z) \cdots G_{M-1}(z)\right]^{t}$, where $G_{k}(z)$ may or may not be related as in (101). Let $X(z)$ denote the system input and let $\boldsymbol{Y}(z)=\left[Y_{0}(z) \cdots\right.$ $\left.Y_{M-1}(z)\right]^{\prime}$ be the system outputs. Now consider the system shown in Fig. 24. Note that

$$
\boldsymbol{G}_{N-1}(z)=\left[\begin{array}{c}
G_{0}(z) \\
\vdots \\
G_{M-1}(z)
\end{array}\right]=\frac{\boldsymbol{Y}(z)}{X(z)}=\mathscr{T}(z)\left[\begin{array}{c}
P_{0} \\
Q_{0} \\
\vdots \\
S_{0}
\end{array}\right]
$$

hence

$$
\left[\begin{array}{c}
X_{0}(z) \\
\vdots \\
X_{M-1}(z)
\end{array}\right]=\mathscr{T}^{t}\left(z^{-1}\right) \mathscr{T}(z)\left[\begin{array}{c}
P_{0} \\
Q_{0} \\
\vdots \\
S_{0}
\end{array}\right] X(z)
$$

which results in

$$
\hat{X}(z)=\left[\begin{array}{lll}
P_{0} & Q_{0} \cdots & S_{0}
\end{array}\right] \mathscr{T}^{t}\left(z^{-1}\right) \mathscr{T}(z)\left[\begin{array}{c}
P_{0} \\
Q_{0} \\
\vdots \\
S_{0}
\end{array}\right] X(z) .
$$

Because of the LBR property of $\mathscr{T}(z)$ and in view of (85), we get

$$
\hat{X}(z)=X(z) .
$$




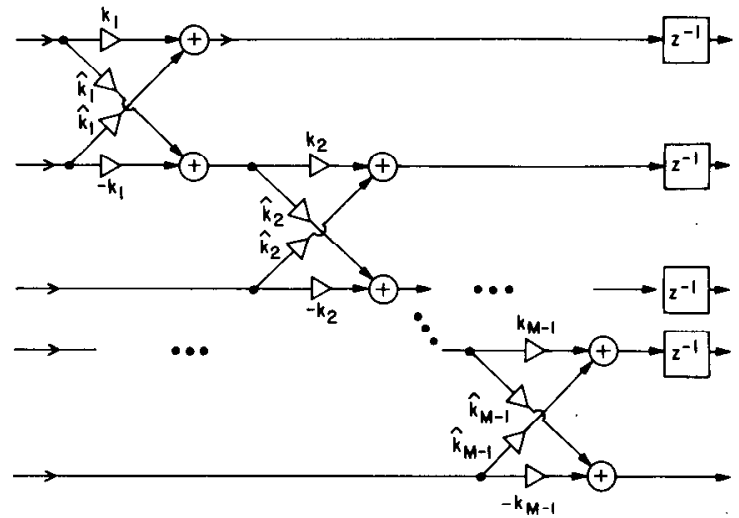

Fig. 25. The building blocks involved in $z^{-(N-1) \mathscr{T}^{t}}\left(z^{-1}\right)$.

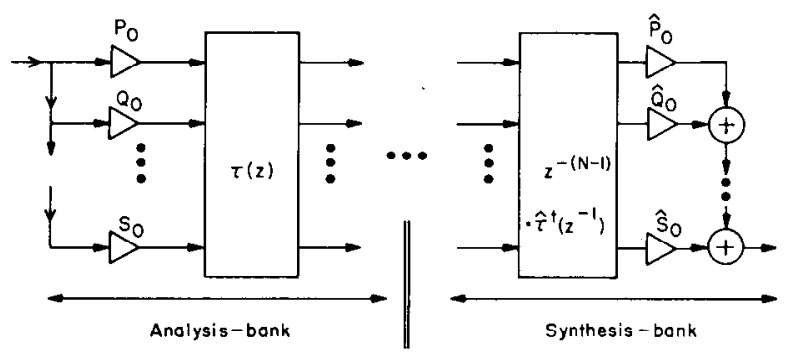

Fig. 26. The modified analysis-synthesis filter bank.

Thus, Fig. 24 represents a perfect analysis-synthesis signal reconstruction system, primarily because of the LBR properties indicated. Notice that $\mathscr{T}^{t}\left(z^{-1}\right)$ is noncausal, and in practice we need to use $z^{-(N-1) \mathscr{T}^{t}\left(z^{-1}\right)}$ in order to obtain a realizable system. Thus, $\hat{x}(n)=x(n-N+1)$, in a practical circuit of this type.

If the set of functions $G_{k}(z)$ happen to be related as in (101) (uniform filter bank), then the simplified version shown in Fig. 23 can be used in the analysis bank, and the corresponding transpose structure in the synthesis bank.

\section{B. Further Simplification of the Analysis-Synthesis Bank of Fig. 24}

Recall that the "analysis bank" of Fig. 24 is a cascade of building blocks as in Fig. 18. Accordingly, $z^{-(N-1) \mathscr{T}^{t}}\left(z^{-1}\right)$ has building blocks of the form shown in Fig. 25, which therefore involves $M-1$ delays per section. Thus, the synthesis bank is highly nonminimal in terms of delays. Recall that, in Section VI, we developed an alternative cascaded lattice structure for vector FIR allpass functions (Figs. 19 and 20). Thus, $\boldsymbol{G}_{N-1}(z)$ can be implemented either as

$$
\boldsymbol{G}_{N-1}(z)=\mathscr{T}(z) \boldsymbol{G}_{0}
$$

or equivalently as

$$
\boldsymbol{G}_{N-1}(z)=\hat{\mathscr{T}}(z) \hat{\boldsymbol{G}}_{0}
$$

where $\boldsymbol{G}_{0}=\left[\begin{array}{lll}P_{0} & Q_{0} \cdots S_{0}\end{array}\right]^{t}$ and $\hat{\boldsymbol{G}}_{0}=\left[\begin{array}{ll}\hat{P}_{0} & \hat{Q}_{0} \cdots \hat{S}_{0}\end{array}\right]^{t}$. The alternative implementation of the analysis-synthesis

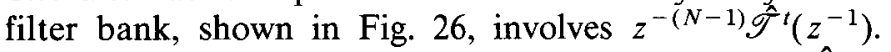
Since the implementation of each section (Fig. 19) in $\hat{\mathscr{T}}(z)$ requires $M-1$ delays, the implementation of each section

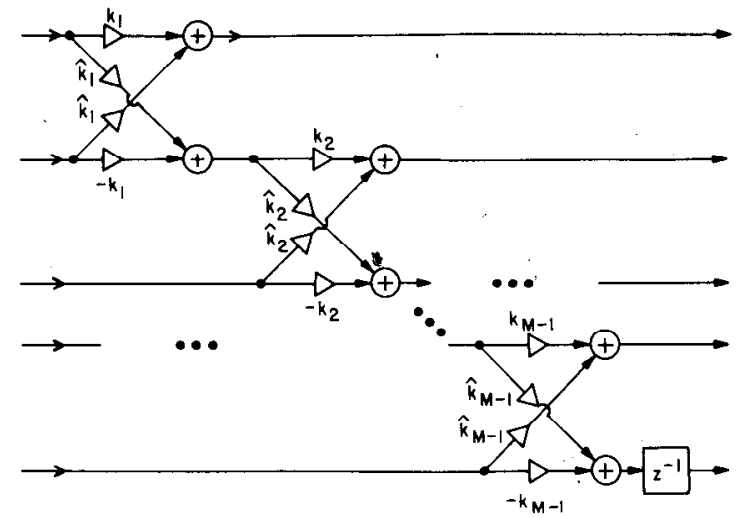

Fig. 27. The building blocks involved in $z^{-(N-1)} \hat{\mathscr{T}}^{t}\left(z^{-1}\right)$.

in $z^{-(N-1) \hat{\mathscr{T}}^{t}}\left(z^{-1}\right)$, shown in Fig. 27, requires only one delay. Thus, the analysis-synthesis bank of Fig. 26 is minimal in terms of delays. Notice that, because of inherent automatic scaling of internal signals, our structures are not minimal in terms of the number of multipliers when compared to direct-form structures.

\section{IX. 'An Application in the Design of QMF (MUlTiRATE) Filter BANKS}

An important class of filter banks used in the subband coding of signals is the quadrature-mirror-filter (QMF) banks [23], [30]. These find application in cases where a signal should be split into several frequency bands and undersampled before coding and transmission, and then suitably recombined at the receiver. A number of versions of such QMF banks are known, some based on IIR filters and some on FIR filters.

A typical filter bank of this type is shown in Fig. 28, for which detailed analyses can be found in [23] and [30]. Here, $H_{0}(z)$ and $H_{1}(z)$ are, respectively, low-pass and high-pass functions, designed to meet certain attenuation requirements in their respective stopbands. In general, one can always find combinations of $H_{0}(z), H_{1}(z), G_{0}(z)$, and $G_{1}(z)$ such that $\hat{X}(z)$ is free from aliasing errors. With such combinations, $\hat{X}(z)$ is then related to $X(z)$ by means of a transfer function

$$
\hat{X}(z)=T(z) X(z) .
$$

Thus, the reconstructed signal $\hat{x}(n)$ is a distorted version of $x(n)$. If $T(z)$ is an IIR allpass function, then we have only phase distortion, while if $T(z)$ is a linear-phase FIR function, we have only amplitude distortion.

Smith and Barnwell have shown [30] that, if $H_{0}(z)$ and $H_{1}(z)$ are restricted to be FIR, but not constrained to be linear-phase FIR, then the circuit of Fig. 28 can be suitably designed such that, not only is the aliasing error perfectly canceled, but, in addition, $T(z)$ becomes a simple delay. In other words, $\hat{x}(n)$ is an exact replica of $x(n)$ with no amplitude or phase distortion, except for a fixed amount of time delay. The precise choice of the relation between the transfer functions $H_{0}(z), H_{1}(z), G_{0}(z)$, and 


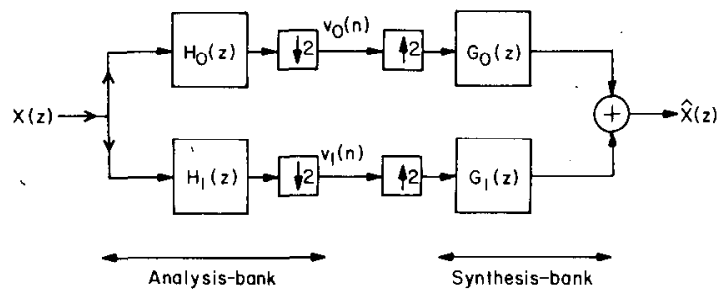

Fig. 28. The quadrature-mirror filter bank.

$G_{1}(z)$ that meets these objectives is given by

$$
\begin{array}{ll}
H_{1}(z)=z^{-(N-1)} H_{0}\left(-z^{-1}\right), & G_{0}(z)-z^{-(N-1)} H_{0}\left(z^{-1}\right) \\
G_{1}(z)=H_{0}(-z) & \text { (117) }
\end{array}
$$

which leads to perfect cancellation of the aliasing term and hence

$$
\begin{aligned}
\hat{X}\left(e^{j \omega}\right)= & \frac{1}{2} e^{-j(N-1) \omega} \\
& \cdot\left[\left|H_{0}\left(e^{j \omega}\right)\right|^{2}+\left|H_{0}\left(-e^{j \omega}\right)\right|^{2}\right] X\left(e^{j \omega}\right) .
\end{aligned}
$$

Or, in terms of $H_{1}(z)$

$$
\hat{X}\left(e^{j \omega}\right)=\frac{1}{2} e^{-j(N-1) \omega}\left[\left|H_{0}\left(e^{j \omega}\right)\right|^{2}+\left|H_{1}\left(e^{j \omega}\right)\right|^{2}\right] X\left(e^{j \omega}\right) .
$$

It is possible to design nonlinear-phase FIR filters $H_{0}(z)$ and $H_{1}(z)$ such that

$$
H_{1}(z)=z^{-(N-1)} H_{0}\left(-z^{-1}\right)
$$

and

$$
\left|H_{0}\left(e^{j \omega}\right)\right|^{2}+\left|H_{1}\left(e^{j \omega}\right)\right|^{2}=1
$$

as shown in [30]. Such designs, therefore, lead to perfect reproduction of the signal $x(n)$ in Fig. 28, except for an overall delay to ensure causality of building blocks. It should be noticed that, unlike the structure of Fig. 28, the original circuit in [30] contains an additional delay operator and an additional advance operator. A causal version of this circuit, along with appropriate redefinitions of $H_{1}(z), G_{0}(z)$, and $G_{1}(z)$ leads to Fig. 28 . The choice in (117) is merely a causal version of the method outlined in [30].

Now in view of (120), the analysis filter bank in Fig. 28 can be most conveniently implemented in the form of the cascaded lossless FIR lattice structure of Fig. 3. A numerical example is presented next.

\section{Design Example 3}

The transfer functions $H_{0}(z)$ and $H_{1}(z)$ satisfying (119) and (120) simultaneously are obtained by following the procedure outlined in [30]. Specifically, for our numerical example, we first invoke the McClellan-Parks algorithm [27] to obtain a zero-phase low-pass FIR transfer function $R_{0}(z)$ of order $2(N-1)$, and having specifications that are

\begin{tabular}{|c|c|c|c|c|}
\hline $\mathrm{m}$ & $k_{m}$ & $\hat{k}_{m}$ & $\alpha_{m}$ & $h_{0}(m)$ \\
\hline $\begin{array}{l}0 \\
1 \\
2 \\
3 \\
4 \\
5 \\
6 \\
7 \\
8 \\
9 \\
10 \\
11 \\
12 \\
13 \\
14 \\
15 \\
16 \\
17 \\
18 \\
19\end{array}$ & $\begin{array}{r}-0.707097 \\
-0.348853 \\
1.000000 \\
0.753874 \\
1.000000 \\
-0.896048 \\
1.000000 \\
0.951180 \\
-1.000000 \\
-0.975958 \\
-1.000000 \\
0.988218 \\
-1.000000 \\
-0.994523 \\
1.000000 \\
0.997696 \\
1.000000 \\
-0.999148 \\
1.000000 \\
-0.027714\end{array}$ & $\begin{array}{l}0.707117 \\
0.937177 \\
0 . \\
0.657019 \\
0 . \\
0.443958 \\
0 . \\
0.308638 \\
0 . \\
0.217959 \\
0 . \\
0.153050 \\
0 . \\
0.104517 \\
0.0 \\
0.067843 \\
0.041279 \\
0.041279 \\
0 . \\
0.999616\end{array}$ & $\begin{array}{c}0.99997 \\
-0.37224 \\
0.07152 \\
0.87152 \\
0 . \\
-0.49546 \\
0 . \\
0.32448 \\
0 . \\
-0.22333 \\
0 . \\
0.15487 \\
0 . \\
-0.10509 \\
0.06800 \\
0.0680 \\
0.0 \\
-0.04131 \\
0.02772 \\
-0.02772\end{array}$ & $\begin{array}{r}0.15149 \\
0.40694 \\
0.46489 \\
0.16371 \\
-0.15944 \\
-0.13227 \\
0.07785 \\
0.09285 \\
-0.04738 \\
-0.06289 \\
0.03285 \\
0.04068 \\
-0.02403 \\
-0.02443 \\
0.01746 \\
0.01314 \\
-0.01215 \\
-0.00670 \\
0.01131 \\
-0.00420\end{array}$ \\
\hline
\end{tabular}
symmetric with respect to $\pi / 2$ : passband edge $\omega_{p}=0.4 \pi$, stopband edge $\omega_{s}=0.6 \pi$, and order $2(N-1)=38$. The symmetry of the response of $R_{0}(z)$ around $\omega=\pi / 2$ ensures
TABLE II

LATTICE COEFFICIENTS AND $h_{0}(m)$ FOR EXAMPLE 3 (Values shown are unquantized)

that the following condition holds ${ }^{4}$

$$
R_{0}(z)+R_{0}(-z)=\text { constant }
$$

Next, a zero-phase low-pass FIR transfer function $F_{0}(z)$ is contructed from $R_{0}(z)$ such that $F_{0}\left(e^{j \omega}\right)$ is nonnegative

$$
F_{0}(z)=R_{0}(z)+\delta \text {. }
$$

$F_{0}(z)$ can therefore be spectral-factorized

$$
F_{0}(z)=H_{0}(z) H_{0}\left(z^{-1}\right)
$$

where $H_{0}(z)$ is of order $N-1$, and has real coefficients. Moreover, in view of $(121), F_{0}(z)$ satisfies

$$
F_{0}(z)+F_{0}(-z)=\text { constant. }
$$

The constant in (123b) can be made equal to unity trivially by scaling. Thus, if $H_{1}(z)$ is defined as in (117), then in view of (123), eq. (120) automatically holds. Specifically, for the purposes of our numerical example, we take $H_{0}(z)$ to be the minimum-phase spectral factor of (123a) and then define

$$
\begin{aligned}
& H_{1}(z)=z^{-19} H_{0}\left(-z^{-1}\right), G_{0}(z)=z^{-19} H_{0}\left(z^{-1}\right) \\
& G_{1}(z)=H_{0}(-z)
\end{aligned}
$$

so that, referring to Fig. 28

$$
\hat{X}(z)=\frac{1}{2} z^{-19} \cdot X(z) .
$$

The FIR allpass vector $\boldsymbol{H}_{a}(z)=\left[\begin{array}{lll}H_{0}(z) & z^{-19} H_{0}\left(-z^{-1}\right)\end{array}\right]^{t}$ is now implemented as a cascaded lossless lattice, as in Fig. 3. This, then, completes the "analysis bank" design in Fig. 28. The "synthesis bank" vector $\boldsymbol{H}_{S}(z)$ in Fig. 28 is

$$
\boldsymbol{H}_{S}(z)=\left[\begin{array}{c}
z^{-19} H_{0}\left(z^{-1}\right) \\
H_{0}(-z)
\end{array}\right]=z^{-19} \boldsymbol{H}_{a}\left(z^{-1}\right) .
$$

\footnotetext{
${ }^{4}$ Notice that, if $N-1$ is even then, because of (121), the coefficient of $R_{0}(z)$ corresponding to $z^{-(N-1)}$ is zero. Hence, $N-1$ can always be taken to be odd, without loss of generality.
} 


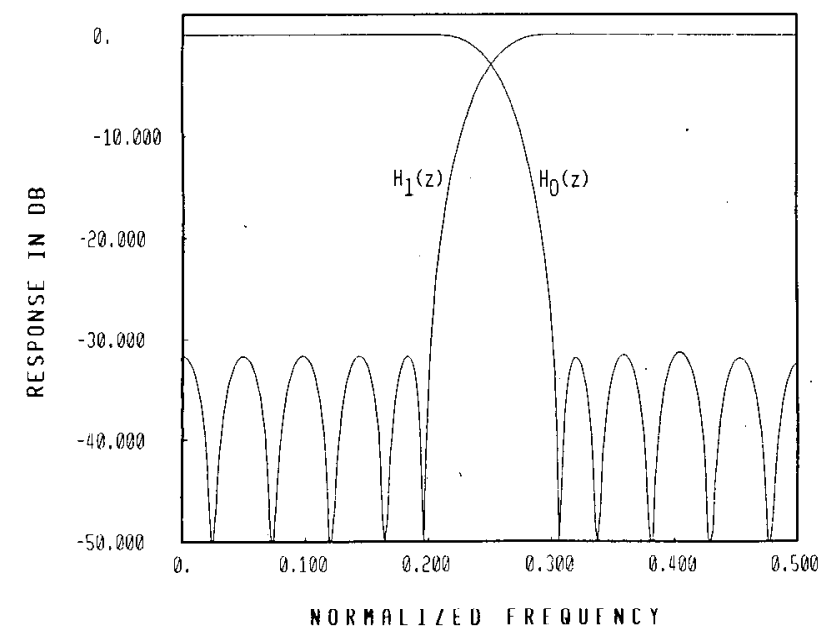

Fig. 29. Example 3. Responses of the lattice-simulated QMF analysis filters.

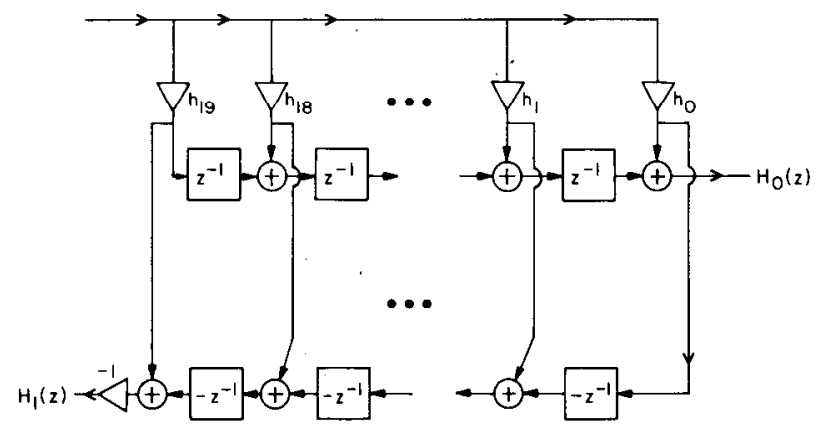

Fig. 30. $\Lambda$ direct-form implementation of the analysis filters $H_{0}(z)$ and $H_{1}(z)$ with 20 multipliers.

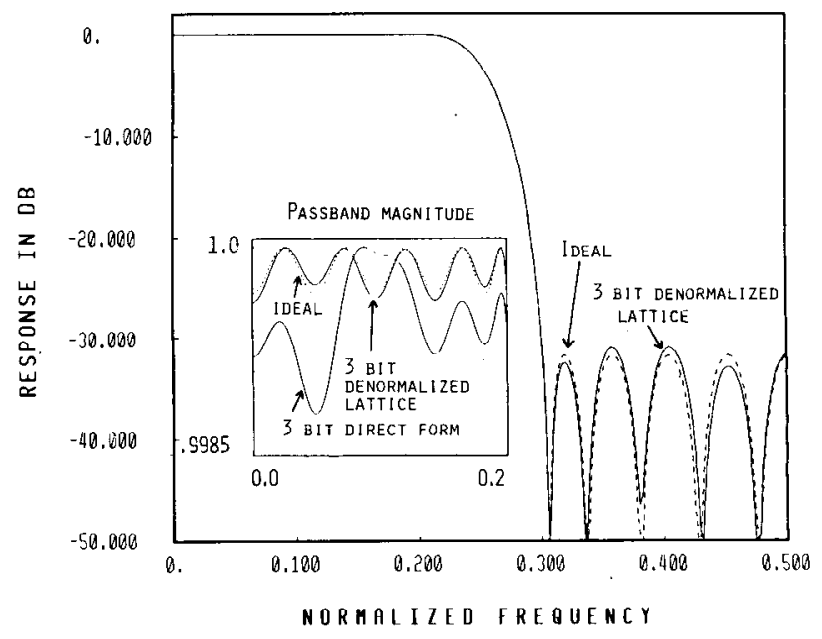

(a)

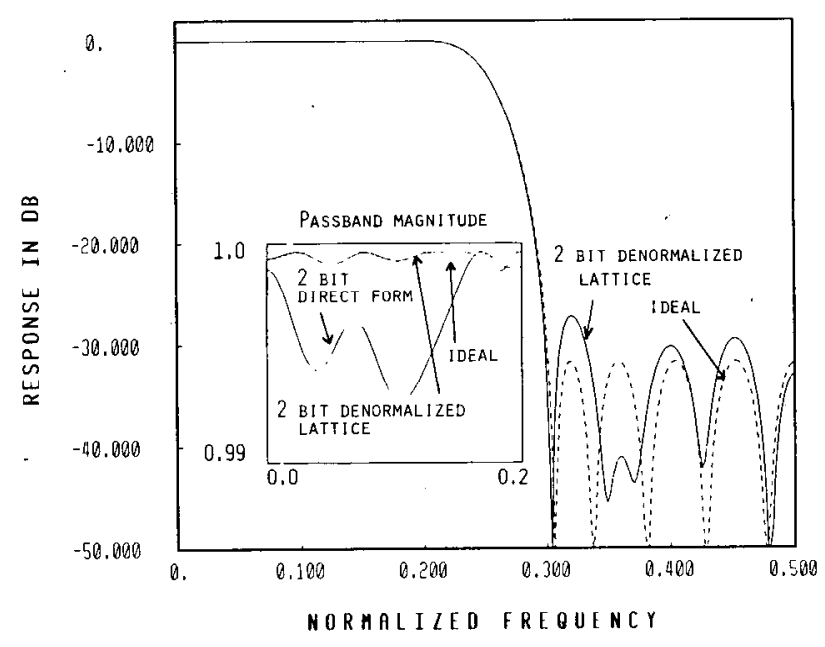

(b)

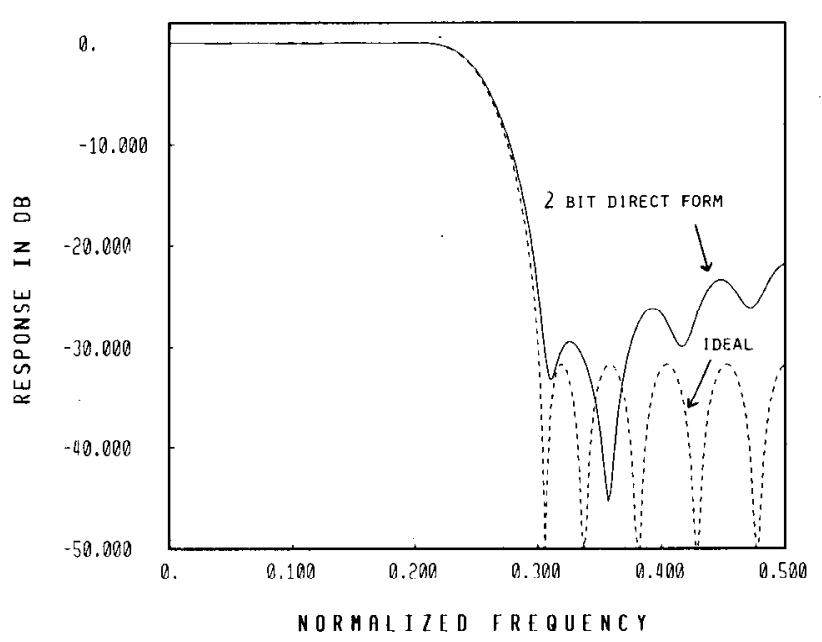

(c)

Fig. 31. (a) Example 3. Responses with 3-bit quantization. (b) Example 3. Responses with 2-bit quantization. (c) Example 3. Response of the 2-bit direct form structure. 


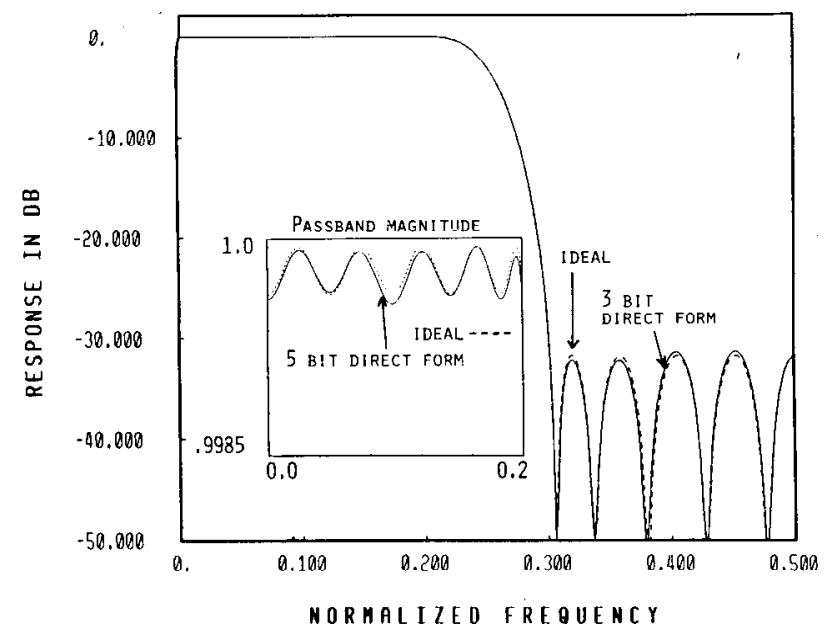

Fig. 32. Example 9.1. Response of the direct-form structure. The passband response is close to ideal if 5 bits are used per coefficient. The stopband response is satisfactory even with 3 bits.

TABLE IV

PerformanCe Summary For Example 3

\begin{tabular}{c|c|c|c|c}
\hline $\begin{array}{c}\text { FIR } \\
\text { Structure }\end{array}$ & $\begin{array}{c}\text { Total Number } \\
\text { of Multipliers In } \\
\text { Analyais Bank }\end{array}$ & $\begin{array}{c}\text { Number of } \\
\text { Bits per } \\
\text { Multiplier }\end{array}$ & $\begin{array}{c}\text { Bit-Multiplier } \\
\text { Product }\end{array}$ & $\begin{array}{c}\text { Number } \\
\text { of Delays }\end{array}$ \\
\hline Direct Form & 19 & 5 & 95 & 38 \\
$\begin{array}{c}\text { Denormalised } \\
\text { Lattice }\end{array}$ & 20 & 3 & 60 & 19 \\
\hline
\end{tabular}

It can be shown that, with $H_{0}(z)$ and $H_{1}(z)$ related as in (119) and (120), the lattice coefficients in Fig. 3 satisfy the property

$$
k_{0}^{2}=\hat{k}_{0}^{2}-1 / 2, \quad k_{2 m}^{2}=1, \quad m \neq 0 .
$$

Thus, the even-numbered lattice sections are multiplierless. In view of this, and in view of the fact that the lattice structure automatically satisfies (120), we feel that these structures are particularly well suited for this particular subclass of QMF banks. The odd-numbered lattice sections can be implemented in denormalized form (Fig. 4) if it is necessary to minimize multipliers. Table II shows the lattice coefficients, and the denormalized multiplier-coefficient $\alpha_{m}$. The table also includes the direct-form coefficients of the low-pass transfer function $H_{0}(z)$ (which is $Q_{N-1}(z)$ according to Fig. 3 notation). Fig. 29 shows the magnitude responses of $H_{0}(z)$ and $H_{1}(z)$.

The total number of multipliers in the denormalized lattice is equal to 20 , for the entire analysis bank. For the direct-form, a structure with 20 multipliers $(19$, if an ovcrall scale factor is ignored) for the analysis bank can be obtained as in Fig. 30, because of (119).

The multipliers $\alpha_{m}$ in the denormalized lattice, quantized to a sum of three powers of two, are shown in Table III, along with the nonzero powers of two in the binary representations. Fig. 31(a) shows the magnitude response of $H_{0}(z)$ with multipliers quantized to 3 bits. The passband details for 3-bit quantization are also shown here. Figs. 31(b) and (c) show the lattice structure and the direct-form responses, with 2 bits per multiplier. The quantized lattice has much superior response than the direct-form and essentially resembles the ideal, unquantized response.

In order to obtain a comparison measure based on bit-multiplier products, the direct form was simulated at higher precisions. The 5-bit direct-form was found to have a performance comparable to the 3-bit latticc (Fig. 32). Table IV summarizes the results, including bit-multiplier products.

\section{Concluding Remarks}

In this paper, a procedure has been outlined for the synthesis of a cascaded-lattice structure that can realize an arbitrary $M$-component FIR allpass transfer function $\boldsymbol{G}_{N-1}(z)$. The structure is in the form of a nonrecursive cascade of lossless, nonrecursive building blocks. Each building block is either a planar rotator or a succession of planar rotators. The structures are automatically internally scaled in an $L_{2}$ sense. These structures are passive and find applications in low-sensitivity FIR filter implementations and in the implementation of filter banks, including quadrature mirror filter implementations for multirate signal processing. Some of these applications were demonstrated in the paper by numerical design examples.

\section{REFERENCES}

[1] A. Fettweis, "Digital filter structures related to classical filter networks," Arch. Elek. Übertrangung., vol. 25, pp. 79-89, Feb. 1971.

[2] A. G. Constantinides, "Alternative approach to design of wave digital filters," Electron. Lett., vol. 10, pp. 59-60, 1974.

[3] J. Szczupak and S. K. Mitra, "On digital filter structures with low coefficient sensitivities," Proc. IEEE, vol. 66, pp. 1082-1083, Sept. 1978.

[4] P. DeWilde and E. Deprettere, "Orthogonal cascade realization of real multiport digital filters," Int. J. Circuit Theory Appl., vol. 8, pp. $245-277,1980$

[5] S. K. Rao and T. Kailath, "Orthogonal digital filters for VLSI implementation," IEEE Trans. Circuits Syst., vol. CAS-31, pp. 933-945, Nov. 1984.

[6] A. H. Gray, Jr., and J. D. Markel, "A normalized digital filter structure," IEEE Trans. Acoustics, Speech, Signal Process., vol. ASSP-23, pp. 268-277, June 1975.

[7] A. H. Gray, Jr., "Passive cascaded lattice digital filters," IEEE Trans. Circuits Syst., vol. CAS-27, pp. 337-344, May 1980

[8] D. A. Vaughan-Pope and L. T. Bruton, "Transfer function synthesis using generalized doubly terminated two-pair networks," IEEE Trans. Circuits Syst, vol. CAS-24, pp. 79-88, Feb. 1977.

[9] Y. C. Lim and S. R. Parker, "On the synthesis of lattice parameter digital filters," IEEE Trans. Circuits Syst., vol. CAS-31, pp. 593-601, July 1984.

[10] J. Makhoul, "Stable and efficient lattice methods for linear prediction," IEEE Trans. Acoustics, Speech, Signal Process., vol. ASSP25, pp. 423-428, Oct. 1977.

[11] J. D. Markel and A. H. Gray, Jr., Linear Prediction of Speech. New York: Springer-Verlag, 1976.

[12] A. Fettweis, "Pseudopassivity, sensitivity, and stability of wave digital filters," IEEE Trans. Circuit Theory, vol. CT-19, pp. 668-673, Nov. 1972.

[13] A. Fettweis and K. Meerkotter, "Suppression of parasitic oscillations in wave filtcrs," IEEE Trans. Circuits Syst., vol. CAS-22, pp. 239-246, Mar. 1975.

[14] P. P. Vaidyanathan, "A unified approach to orthogonal digital filters and wave digital filters, based on LBR two-pair extraction," IEEE Trans. Circuits Syst., vol. CAS-32, pp. 673-686, July 1985.

[15] O. Herrmann and W. Schuessler, "On the accuracy problem in the design of nonrecursive digital filters," Arch. Elek. Übertragung., vol. 24, pp. $525-526,1970$.

[16] W. Schuessler, "On structures for nonrecursive digital filters," Arch. Elek. Übertragung., vol. 26, pp. 255-258, 1972. 
[17] D. S. K. Chan and L. R. Rabiner "Analysis of quantization errors in the direct form for finite impulse response digital filters," IEEE Trans. Audio Electroacous, vol. AU-21, pp. 354-366, Aug. 1973.

[18] D. S. K. Chan, "Roundoff noise in cascade realization of finite impulse response digital filters," M. S. thesis, Dept. Elec. Eng., MIT, June 1972.

[19] A. V. Oppenheim and R. W. Schafer, Digital Signal Processing. Englewood Cliffs, NJ: Prentice-Hall, 1975.

[20] L. R. Rabiner and B. Gold, Theory and Application of Digital Signal Processing. Englewood Cliffs, NJ: Prentice-Hall, 1974.

[21] J. Makhoul "A class of all-zero lattice digital filters: properties and applications," IEEE Trans. Acoustics, Speech, Signal Process., vol. ASSP-26, pp. 304-314, Aug. 1978.

[22] D. M. Goodman and E. K. Miller, "A note on minimizing the prediction error when the zeros are restricted to the unit circle," IEEE Trans. Acoustics, Speech, Signal Process., vol. ASSP-30, pp. 503-505, June 1982.

[23] R. F. Crochiere and I. R. Rabiner, Multirate Digital Signal Processing. Englewood Cliffs, NJ, Prentice-Hall, 1983.

[24] P. P. Vaidyanathan and S. K. Mitra, "Low passband sensitivity digital filters: A generalized viewpoint and synthesis procedures," Proc. IEEE, vol. 72, pp. 404-423, Apr. 1984

[25] P. P. Vaidyanathan and S. K. Mitra, "Passivity properties of low sensitivity digital filter structures," IEEE Trans. Circuits Syst., vol. CAS-32, pp. 217-224, Mar. 1985

[26] G. A. Mian and A. P. Nainer, "A fast procedure to design equiripple minimum-phase FIR filters," IEEE Trans. Circuits Syst., vol. CAS-29, pp. 327-331, May 1982 .

[27] J. H. McClellan, T. W. Parks, and L. R. Rabiner, "FIR linear-phase filter design program," in Programs for Digital Signal Processing. New York: IEEE Press, 1979.

[28] P. P. Vaidyanathan, "The discrete-time bounded real lemma in digital filtering," IEEE Trans. Circuits Syst., vol. CAS-32, pp. $918-924$, Sept. 1985

[29] M. G. Bellanger, G. Bonnerot, and M. Coudreuse, "Digital filtering by polyphase network: Application to sample-rate alteration and filter banks," IEEE Trans. Acoustics, Speech, Signal Process., vol. ASSP-24, pp. 109-114, Apr. 1976.
[30] M. J. T. Smith and T. P. Barnwell III "A procedure for designing exact reconstruction filter banks for tree-structured subband coders," in IEEE Int. Conf. Acoustics, Speech, Signal Process., (San Diego, CA), Mar. 1984, pp. 27.1.1-27.1.4

[31] P. P. Vaidyanathan, "On power-complementary FIR filters," IEEE Trans. Circuits Syst., vol. CAS-32, pp. 1308-1310, Dec. 1985.

[32] A. Fettweis, "Wave digital filters: Theory and practice," Proc. IEEE, vol. 74, pp. 270-327, Feb. 1986.

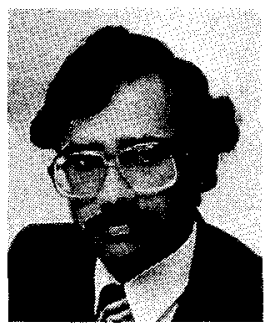

P. P. Vaidyanathan (S'80-M'83) was born in Calcutta, India, on October 16, 1954. He received the B.Sc (with honors) degree in physics, and B. Tech. and $M$. Tech. degrees in radiophysics and electronics from the University of Calcutta, India, in 1974, 1977, and 1979, respectively, and the Ph.D. degree in electrical and computer engineering from the University of California, Santa Barbara, in 1982.

He was a postdoctoral fellow at the University of California, Santa Barbara, from September 1982 to February 1983. Since March 1983, he has been with the California Institute of Technology, Pasadena, as an Assistant Professor of Electrical Engineering. His main research interests are in digital signal processing, linear systems, and filter design. He served as the Vice-Chairman of the Technical Program Committee of the 1983 IEEE International Symposium on Circuits and Systems. 